Check for updates

Cite this: Mater. Chem. Front., 2020, 4, 317

Received 16th September 2019, Accepted 3rd October 2019

DOI: $10.1039 / c 9 q m 00580 c$

rsc.li/frontiers-materials

\title{
The development of mechanoluminescence from organic compounds: breakthrough and deep insight
}

\author{
Yujun Xie $\mathrm{X}^{\mathrm{a}}$ and Zhen Li (D) *ab
}

\begin{abstract}
Organic mechanoluminescence $(\mathrm{ML})$ compounds have experienced breakthrough developments in recent years, with $M L$ being discovered in many kinds of organic compounds. Accordingly, the ML composition is becoming more complicated; complications can be observed from the initial stages of nitrogen discharge to fluorescence, dual emission of fluorescence and phosphorescence and the more novel persistent roomtemperature phosphorescence (RTP) on to photo-sensitive $\mathrm{ML}$, presenting unique luminous properties with more perspectives. Since $M L$ is found to be highly correlated with the molecular configuration and the intermolecular packing style in the aggregate state, the photophysical properties should be analyzed carefully with the consideration of MUSIC (Molecular Uniting Set Identified Characteristic). In this review paper, we summarized the recently reported organic ML compounds, which were classified according to their luminous and chemical structure features. The investigation of organic ML can promote the development of "aggregated state chemistry" and the luminous theory in the aggregate state.
\end{abstract}

\section{Introduction}

In the past decades, organic luminescent materials have experienced rapid development in both experimental and theoretical aspects and have been widely applied in the fields of organic light-emitting diodes (OLED), biological imaging, environmental protection, etc. ${ }^{1-6}$ Theoretically, luminescence arises from the electron transition from the excited state to the ground state. The identical and opposite electron spin multiplicities between the ground and excited states will lead to different excited states of singlet and triplet states, which radiatively decay as fluorescence and/or phosphorescence. ${ }^{7}$ Despite years of research, the organic luminescence mechanisms are still not fully understood due to the very complicated influence originating from chemical structure, spatial configuration, and ambient environment, etc. Generally, the spin-orbital coupling (SOC) coefficient of organic molecules is small because of the spin-forbidden transition between singlet and triplet states. As a result, the radiative decay rate of triplet excitons is far less than that of non-radiative, and triplet excitons can be easily quenched by oxygen in the air. Therefore, it is difficult to observe phosphorescence in purely organic luminophores at room temperature. However, triplet states have fantastic impacts on luminescence properties since their interplay with singlet states leads to lots

${ }^{a}$ Institute of Molecular Aggregation Science, Tianjin University, Tianjin, 300072, China.E-mail: lizhentju@tju.edu.cn, lizhen@whu.edu.cn

${ }^{b}$ Department of Chemistry, Wuhan University, Wuhan, 430072, China of novel luminescence characteristics. For example, the small energy gap between singlet $\left(\mathrm{S}_{1}\right)$ and triplet $\left(\mathrm{T}_{1}\right)$ states can cause effective reverse intersystem crossing (RISC) in thermally activated delayed fluorescence (TADF) materials, ${ }^{8-10}$ whereas the large energy gap between high-lying triplet states $\left(\mathrm{T}_{n}\right)$ and $\mathrm{T}_{1}$ states as well as the close energy gaps between the high-lying singlet $\left(\mathrm{S}_{n}\right)$ and triplet $\left(\mathrm{T}_{n}\right)$ energy levels will result in hybridized local and charge-transfer (HLCT) emissions. ${ }^{11,12}$ However, many new luminescence mechanisms related to interactions between singlet and triplet states remain to be explored.

Apart from the intrinsic electron levels, the external ambient environment is also very important to the luminous properties of organic molecules. In a dilute solution or dispersion in a solid matrix, luminescence arises from individual molecules once they become excited. In the aggregated state, the situation becomes more complicated, and the photophysical properties are quite different from that in a single molecular state. On the one hand, the molecular vibrations and rotations are strictly restricted by the physical space constraint in the aggregated state, which results in a reduced non-radiative transition rate. On the other hand, intermolecular interactions are enhanced due to the decreased intermolecular distances in the aggregated state. Therefore, the organic molecules will exhibit unique luminous properties in the aggregated state under the complicated effects, which has been widely reported in previous research. For example, the H-aggregates cause the blue-shift in absorption and red-shift in fluorescence but with 
reduced fluorescence efficiency, while the J-aggregates lead to a red-shift in absorption and fluorescence but with increased fluorescence efficiency. ${ }^{13}$ In 2001, Ben Zhong Tang et al. coined the concept of "Aggregation-Induced Emission (AIE)", revealing that molecules with distorted spatial configurations show weak emission in the solution state but intense emission in the aggregated state. ${ }^{14}$ In recent years, organic room temperature phosphorescence (RTP), which is usually observed in molecular aggregates, has been undergoing booming development, ${ }^{15}$ and even some organic cocrystals have been reported with extraordinary properties in light emission, ferroelectricity, optical waveguide and stimuli responsiveness due to noncovalent intermolecular interactions. ${ }^{16-18}$ The photophysical properties in the aggregated state are highly correlated with the molecular structure and arrangement. Accordingly, in 2018, our group proposed the concept of "Molecular Uniting Set Identified Characteristic (MUSIC)" to describe unique photophysical characteristics in aggregated states. ${ }^{19}$ Although the research on chemical properties in the aggregated state is still in its infant stage, the "aggregated state chemistry" has exhibited a significant impact on the structure-property relationships in many fields, such as organic solar cells, organic field-effect transistors, non-linear optics, liquid crystals, etc. Even at the molecular level, Cao et al. reported that stacking two layers of graphene at a "magic angle" difference of about $1^{\circ}$ regulated the carrier concentration through the gate voltage to achieve an insulator with a half-full band, which then achieved a $1.7 \mathrm{~K}$ superconductivity. ${ }^{20,21}$ The investigation of the chemical properties in aggregates could actually be the research hotspot of the future.

Mechanoluminescence (ML), also known as triboluminescence (TL), is a unique property of molecular aggregates, which refers to the phenomenon of light being produced upon the application of force to compounds (Fig. 1). ${ }^{22-27} \mathrm{ML}$ is an ancient luminous phenomenon that dates back to 1605 when Francis Bacon observed a flash of light from hard sugar upon being scraped. ${ }^{22,28,29}$ It has now drawn great attention due to its potential applications in the fields of force sensors, lighting, displays, damage detections, bioimaging and phototherapy, etc. $^{30-39}$ So far, various kinds of compounds have been found

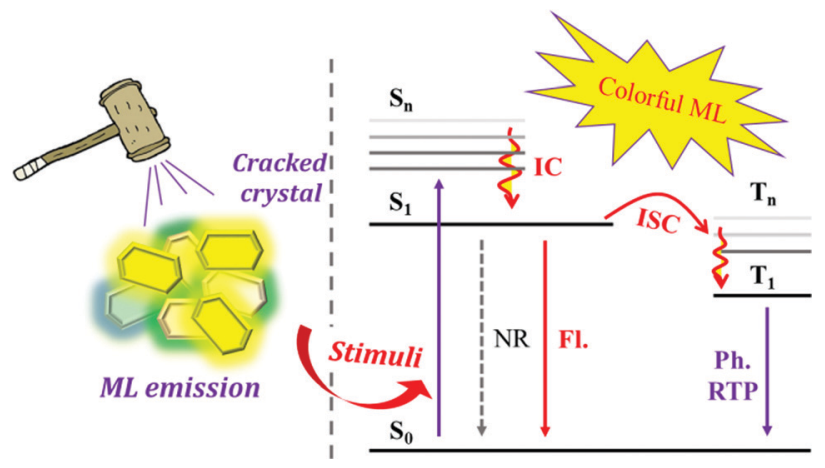

Fig. 1 Schematic representation of mechanoluminescence (ML) in colorful fluorescence (Fl.) and phosphorescence (Ph.), while the mechanical stimuli serve as the excitation source. ISC: intersystem crossing, NR: non-radiative transition, RTP: room-temperature phosphorescence. with ML properties, such as pure organic and inorganic crystals, metal-centred organic chelates, alkali halides, rare-earth-doped ceramics, ZnS crystals, semiconductors, polymers, metals and so on. ${ }^{24,40,41}$ Accordingly, different ML mechanisms of these compounds have been proposed. ${ }^{22,24}$ Although the mechanism of how mechanical force is transformed into light in organic compounds is still not fully understood, the crystal disorder, special molecular packing and piezoelectric effect are usually considered to play key roles in the production of excited states for light emission..$^{22,42-44}$ In detail, the piezoelectric effect results from the acentric space group of crystals, which creates two opposite charge distributions across the crystal, producing an electric field that excites light when the crystal breaks. For organic non-aromatic compounds and some inorganic crystals, such as sucrose, tartaric acid, $\mathrm{Li}_{2} \mathrm{SO}_{4}$, etc., the electric field is strong enough to break down the air and cause nitrogen discharge. ${ }^{22}$ In organic aromatic compounds, the electric field would act as the excitation source to produce ML similar to the corresponding PL. However, there are also some cases of ML from centrosymmetric crystals, and Dickinson et al. concluded that the ML was excited by the electrons, ions, and neutral species that are produced from the fractured surface. ${ }^{45-47}$ Overall, ML is more prone to being observed in acentric crystals. According to statistics, ML occurs more frequently in crystals belonging to the acentric space group than those with the centric space group, ${ }^{44}$ and the MUSIC concept can be applied to the description of ML in organic compounds.

Thanks to the rapid development of experimental techniques and photophysical theory, colorful ML that is closely associated with the molecular configuration and singlet/triplet energy levels has been observed in organic compounds. However, the ML exhibited very different luminous characters in comparison with the corresponding photoluminescence (PL), and the mechanical force stimuli serve as different excitation modes as compared to light. For example, the ML composition could be fluorescence, phosphorescence, or a mixture of these and the emission wavelength or the intensity of the ML spectra would change as well, which might be different from their PL. Although there have recently been some review papers summarizing the research progress, ${ }^{25,27,48}$ the emerging ML compounds exhibit new characteristics and research prospects, which need to be discussed from a broader perspective. Herein, partially based on our systematic work on ML luminogens, we have collected recently published ML molecules, summarized their characteristics, and explored the inherent mechanisms. For the sake of better discussion, we have classified the ML compounds according to their luminous properties and chemical structural features as follows: compounds with ML in the form of fluorescence; ML from AIE compounds and especially the TPE derivatives; molecular configuration-related ML luminogens; compounds with ML consisting of the dual emission of fluorescence and phosphorescence.

Overall, the ML compounds exhibited mechanically-induced fluorescence, phosphorescence, and long lifetime phosphorescence, indicating the MUSIC feature. 


\section{Compounds with $M L$ in the form of fluorescence}

At room temperature, purely organic compounds usually emit fluorescence under light excitation, while phosphorescence is negligible due to the spin forbidden rule. Despite the different excitation modes of mechanical stimuli as compared to photo stimuli, the photophysical process of light emission is almost the same. Therefore, mechanofluorescence is the most common emission type in ML. Actually, all the reported organic ML compounds exhibited fluorescence in ML spectra, and the ML color is mostly blue or green, probably because of the relatively small degree of conjugation and high crystallinity of these molecules. Generally, the mechanofluorescent molecules usually exhibit strong photofluorescence at room temperature. In this section, we summarize some prominent compounds with ML in the form of fluorescence.

It has been reported that some organic non-aromatic compounds like sugars, tartaric acid, etc., can produce ultra-violet (UV) and deep-blue emission under mechanical stimuli. ${ }^{29,49}$ However, their ML was proved to be generated by nitrogen discharge under the electric field between the two fracture surfaces. UV luminophores are relatively rare among organic aromatic compounds, and in 2018, Yang et al. reported a unique compound of BP-TPY, which exhibited deep-blue ML located in the UV region with a peak at $372 \mathrm{~nm}$ (Fig. 2A and B). ${ }^{50}$ Upon excitation of a UV lamp at $300 \mathrm{~nm}$, BP-TPY produced emission at around $368 \mathrm{~nm}$, and showed a narrow full width at half-maximum of $35 \mathrm{~nm}$. At $77 \mathrm{~K}$, both fluorescence and phosphorescence were observed (Fig. 2A); thus, the ML spectrum corresponded well to the PL spectrum. The single-crystal analysis revealed that there were two different twisted conformations

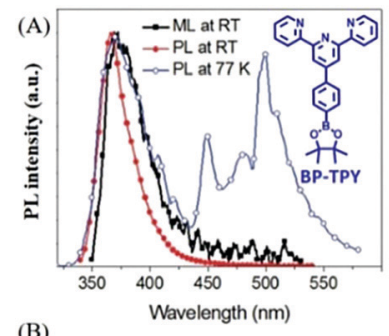

(B)
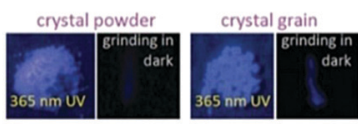
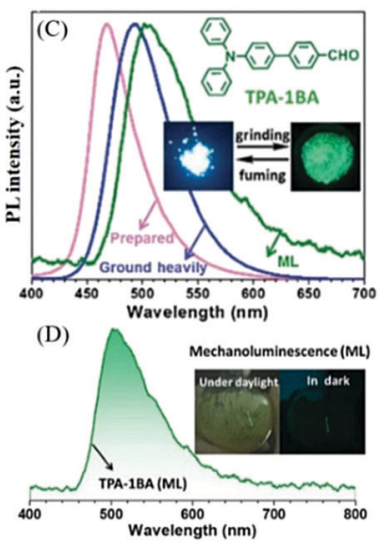

Fig. 2 (A) The ML and PL spectra of BP-TPY crystals at room temperature $(\mathrm{RT})$, and the $\mathrm{PL}$ spectrum recorded at $77 \mathrm{~K}$ under the excitation of a $300 \mathrm{~nm}$ light source. (B) The PL and ML images of crystalline BP-TPY powder and grains at $298 \mathrm{~K}$. Reproduced with permission. ${ }^{50}$ Copyright 2018, Royal Society of Chemistry. (C) PL spectra of TPA-1BA before (asprepared) and after grinding (ground heavily) in the solid-state, and $\mathrm{ML}$ spectrum; photos of TPA-1BA before and after grinding are presented in the inset. (D) ML spectrum of TPA-1BA; inset: the ML photos under daylight and in the dark. Reproduced with permission. ${ }^{51}$ Copyright 2017, Royal Society of Chemistry. present with the formed non-centrosymmetric space group of $P c$ in the monoclinic system, and the UV region ML should be ascribed to the twisted conformation and the small degree of conjugation.

Triphenylamine (TPA) is a common aromatic block in the design of optoelectric functional molecules having a good degree of conjugation and electron-donor properties. The flexible molecular structure and good crystallinity also make TPA derivatives sensitive to the phase transition. Accordingly, the Li group has built a series of TPA derivatives and found some interesting characteristics. In 2017, the benzaldehyde group was introduced to the TPA group as an electron acceptor, and the resultant compound of TPA-1BA ${ }^{51}$ emitted bright green ML that peaked at around $500 \mathrm{~nm}$ under mechanical stimuli (Fig. 2C and D). TPA-1BA also exhibited mechanochromism properties, and the PL of the as-prepared solid red-shifted from 467 to $492 \mathrm{~nm}$ after grinding. The single-crystals of TPA-1BA belong to the piezoelectric non-centrosymmetric space group Pca21, and have good crystallinity and high rigidity. The sharp and intense X-ray diffraction peaks remained after grinding, contributing significantly to the ML properties for the blocking of the possible energyexhausting pathways.

In 2019, Li et al. reported more ML compounds from TPA derivatives with different halogen substituents. ${ }^{52}$ As shown in Fig. 3A, the $\mathrm{F}, \mathrm{Cl}$, and $\mathrm{Br}$ atoms were substituted at the para- or meta-position of the phenyl group of TPA-ph, to yield TPA- $m \mathrm{~F}$, TPA- $m \mathrm{Cl}$, TPA- $m \mathrm{Br}$, TPA- $m \mathrm{~F}$, TPA- $p \mathrm{Cl}$, and TPA- $p \mathrm{Br}$, respectively. The ortho-position substituted derivatives were also obtained but without ML. The ML intensities of these compounds were different: TPA-ph and TPA- $p$ F showed weak ML with the centrosymmetric space group of $P 21 / c 1$, while the strong $\mathrm{ML}$ peaks at 431, 433, 435, 443, and $436 \mathrm{~nm}$ were observed in the crystalline samples of TPA- $m \mathrm{~F}$, TPA- $m \mathrm{Cl}$, TPA- $m \mathrm{Br}$, TPA- $p \mathrm{Cl}$, and TPA- $p$ Br, respectively (Fig. $3 \mathrm{~B}$ and $\mathrm{C}$ ), with the non-centrosymmetric space group of $C 1 c 1$. Their PL properties were also investigated, and TPA-ph without any halogen atoms emitted deep-blue fluorescence at $396 \mathrm{~nm}$. The para-position-substituted

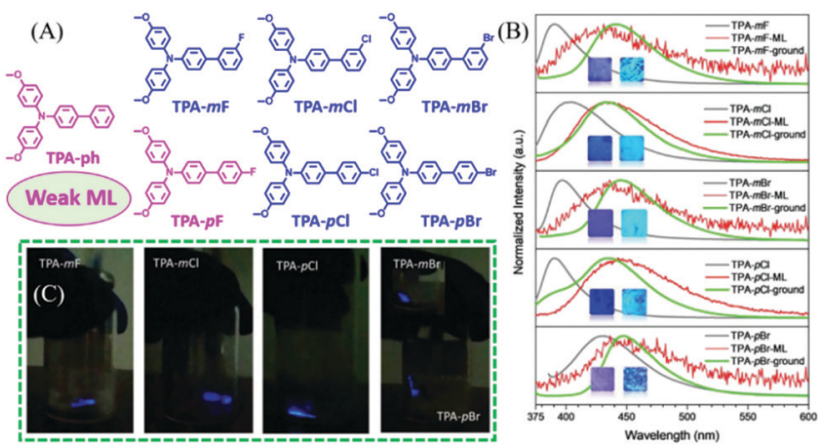

Fig. 3 (A) The molecular structure of TPA-ph, TPA- $m F$, TPA- $m C l$, TPA$m B r$, TPA- $p F$, TPA- $p C l$, and TPA- $p B r$. (B) PL spectra of TPA- $m F$, TPA- $m C l$, TPA- $m B r$, TPA- $p C l$, and TPA- $p B r$ before (gray line) and after (green line) grinding, and the corresponding ML spectra (red line); inset: the photos of as-prepared and ground powder samples under irradiation of $365 \mathrm{~nm}$ UV light. (C) ML images. Reproduced with permission. ${ }^{52}$ Copyright 2019, John Wiley and Sons. 
TPA- $p \mathrm{Br}(429 \mathrm{~nm})$ had greater red-shifted emission as compared to TPA- $p$ F (391 nm) and TPA- $p$ Cl (390 nm), possibly due to the stronger intersystem charge transfer. However, the meta-position substituted TPA- $m$ F (390 nm), TPA- $m$ Cl (403 nm), and TPA- $m \mathrm{Br}$ (397 nm) showed similar emissions at 390, 403, and $397 \mathrm{~nm}$, respectively. As shown in Fig. 3B, high-contrast mechanochromism was observed in all compounds, with the PL redshifting by about $51 \mathrm{~nm}$ after grinding. Thus, all the ML spectra corresponded well with the PL spectra of the ground samples, indicating that the ML was produced from the monomer at the cracked surface of the crystal.

Carbazole is another famous molecular building block in the field of optoelectronic functional materials. It has been reported that the carbazole single-crystal produces $\mathrm{ML}$, but the visible emission was too weak to be recorded by the naked eyes. ${ }^{53}$ Also, the carbazole crystals showed very good RTP features with ultralong lifetimes of $910 \mathrm{~ms}$ at $555 \mathrm{~nm},{ }^{55}$ further demonstrating their very complicated electron interactions. A series of carbazole derivatives also exhibited unique ML (Fig. 4). For example, $N$-isopropylcarbazole (NIPC) had a very strong blue ML that could be observed even under daylight conditions, ${ }^{56}$ and 3,6-dibromocarbazole (DBC) showed dual-modes of $\mathrm{ML}$ composed of weak fluorescence and strong phosphorescence according to the ML spectrum, which can be ascribed to the "heavy atom effect" of two bromine atoms. ${ }^{57} \mathrm{~N}$-Ethyl-3-vinylcarbazole (NE3VCz), 9-isopropyl-3-vinyl-carbazole (NIP3VCz), and 3,9-diethyl-carbazole (DECz) were also reported to possess ML properties. ${ }^{58}$

Interestingly, some carbazole derivatives with extraordinary ML were recently reported. In 2018, Yang et al. reported that strong blue ML was observed in indoor natural light from the lamellar crystal of $N$-phenylcarbazole (NPC, Fig. 5A) upon gently touching with a metal spade, or by violently shaking or stirring with a magnetic bar. ${ }^{53}$ The lump crystal obtained from cooled melting and sublimation could also emit bright blue $\mathrm{ML}$ (Fig. 5C). Although the visible RTP with a lifetime of $13.4 \mathrm{~ms}$ and the low-temperature phosphorescence were observed, the ML was ascribed to fluorescence according to the emission spectrum, for the different excitation modes (Fig. 5A). The NPC crystal belonged to the orthorhombic system with the piezoelectric space group of $F d d 2$. Strong intermolecular interactions were observed in the crystal, which favored the production of ML. Sun et al. further explored NPC as the host matrix to realize multicolor ML. ${ }^{54}$ Due to its potential advantages of high

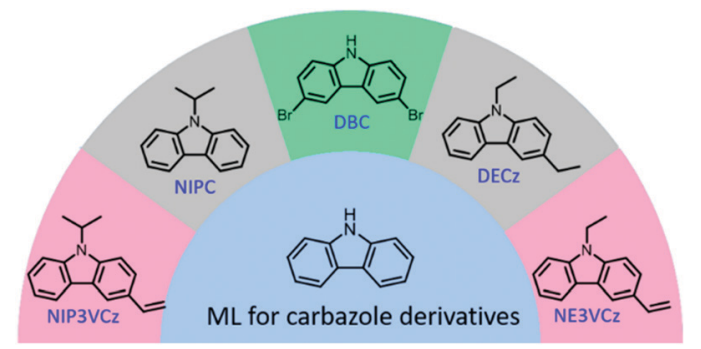

Fig. 4 The reported carbazole derivatives with ML character. thermal stability, and ease of crystallization with a moderate melting point of $91{ }^{\circ} \mathrm{C}$, the bright blue ML of NPC can act as an excitation source. Furthermore, the melted NPC liquid can serve as a non-polar organic solvent for dissolving or dispersing organic fluorescence emitters. Four polar dyes, with green (PPO), yellow (ClDPP), orange (BrDPP) and red (PDPP) PL, were chosen as dopants (Fig. 5F) in amounts of $4-5 \mathrm{w} \%$, by stirring the blends of NPC melting liquid and dyes at $110{ }^{\circ} \mathrm{C}$, followed by cooling to room temperature; their corresponding PL spectra are listed in Fig. 5B. The polar dopants were dispersed in the NPC matrix in the form of small nanocrystals, and the commendable overlaps between the ML spectrum of NPC and the absorption spectra of the dopants (Fig. 5D) promoted the efficient energy transfer between them. The blends exhibited fluorescence and bright green, orange, and red ML due to the presence of dopants (Fig. 5E and F). Thus, this simple strategy provided a feasible approach for realizing bright multicolored ML without difficult molecular designs and syntheses.

Chi et al. reported ML from carbazole derivatives as well. ${ }^{59}$ To achieve repeatable ML in devices for practical applications, they introduced a series of alkyl chains with different lengths at the $N$-position of the carbazole (Cz-alkyl- $N, N=2$ to 8 , in which, $N$ is the number of carbon atoms in the alkyl chain). However, only Cz-alkyl-6 and Cz-alkyl-7 exhibited bright deep blue ML (Fig. 6A and B) similar to their PL spectra. The Cz-alkyl-6 crystal was non-centrosymmetric, while the Cz-alkyl-7 crystal was centrosymmetric. In single crystals, the alkyl chains of these two compounds aggregated and stacked together, and generated alkyl zones with very weak intermolecular interactions, which should be in favor of the sensitive ML for the reduced pressure threshold for ML. Furthermore, both Cz-alkyl-6 and Cz-alkyl-7 had a very strong tendency for crystallization at room temperature and very low melting temperatures of 68.8 and $35.9{ }^{\circ} \mathrm{C}$, respectively. As a result, the polycrystalline samples of both compounds were simply obtained from the hot melting liquids with identical ML and X-ray diffraction (XRD) signals, and the strong emission was also observed during the crystallization process of Cz-alkyl-6 (crystalloluminescence). The ML of the two compounds could be recovered by simply heating, even under solar irradiation, thus making them fascinating solarrenewable organic ML materials (Fig. 6C). More interestingly, colorful organic ML was obtained by doping various fluorescent dyes (the molecular structures are presented in Fig. 6E) with Cz-alkyl-6. The ML properties of the doped system were similar to Cz-alkyl-6, especially the in situ solar-renewable and sensitive ML. As shown in Fig. 6D and F, the ML covered the whole visible region from blue to red with dual emission bands of the host (Cz-alkyl-6) and the guest (fluorescent dyes), and the ML emission color could also be changed by different doping concentrations. The sandwich-type organic ML devices were fabricated by placing the ML sample between quartz and a soft polymer layer. The devices exhibited extremely sensitive ML; for example, the devices doped with $1 \%$ of dye $\mathrm{G}$ emitted obvious green emission upon being lightly touched with fingers or blown with a gentle stream of nitrogen gas. Accordingly, the ML turn-on pressure threshold was measured to be about $5 \mathrm{kPa}$ 

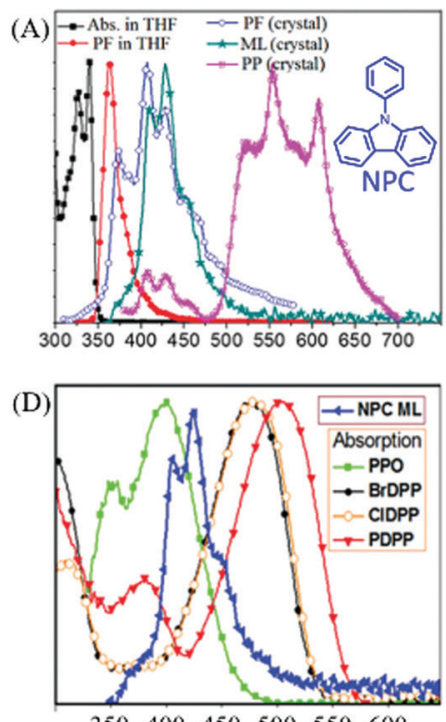

$\begin{array}{lllllll}350 & 400 & 450 & 500 & 550 & 600\end{array}$

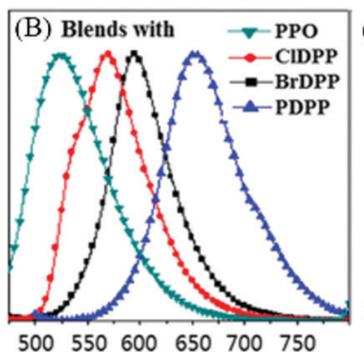

500550600650700750

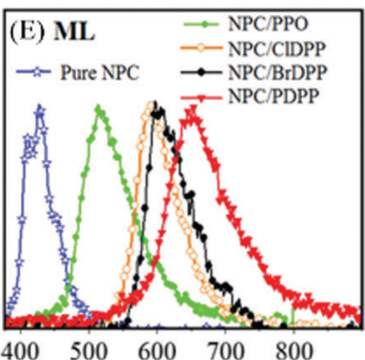

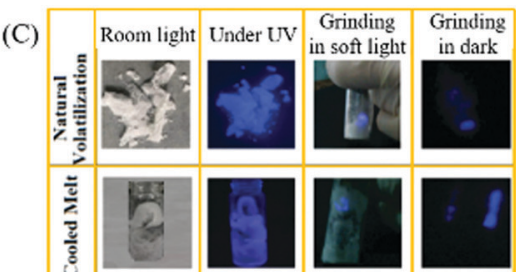

(F)

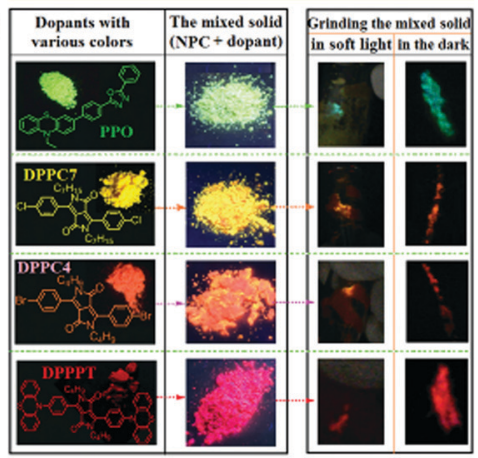

Fig. 5 (A) The absorption (Abs.) and fluorescence (PF) spectra of NPC solution and crystals; ML and RTP (PP) spectra at room temperature; the inset shows the chemical structure of NPC. (B) The PL spectra of NPC blends (4-5 w\%) with PPO, CIDPP, BrDPP and PDPP. (C) ML photos of the NPC samples prepared by natural volatilization and cooled melt under different conditions. (D) The absorption spectra of PPO, CIDPP, BrDPP and PDPP; ML spectrum of NPC. (E) ML spectra of NPC and the blended solids. (F) The molecular structure and photos of PPO, CIDPP, BrDPP and PDPP solids and blends in NPC under ultraviolet irradiation; the ML photos of blends ground in soft light and in the dark. Reproduced with permission. ${ }^{53,54}$ Copyright 2018 , Royal Society of Chemistry.
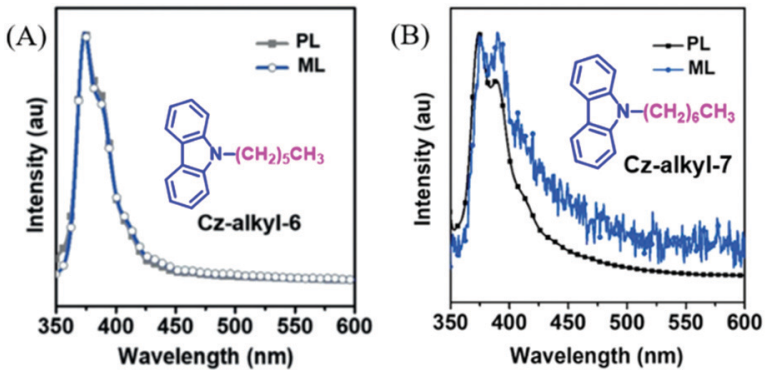

(C)
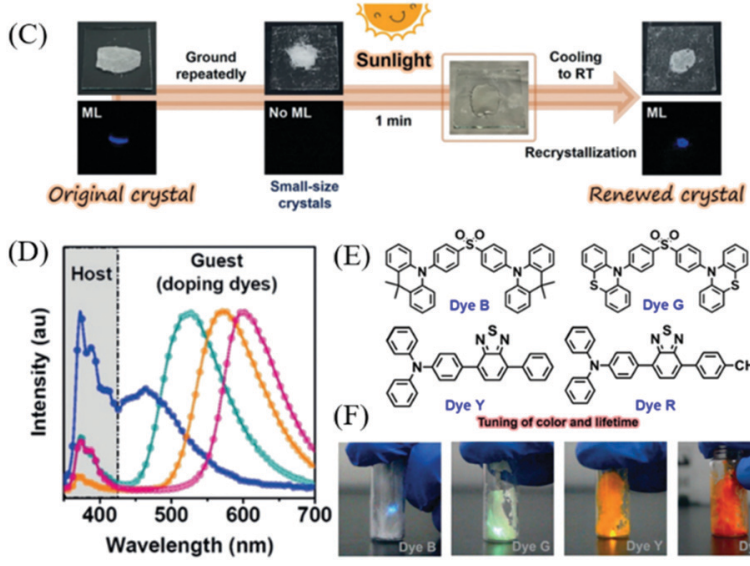

(E)
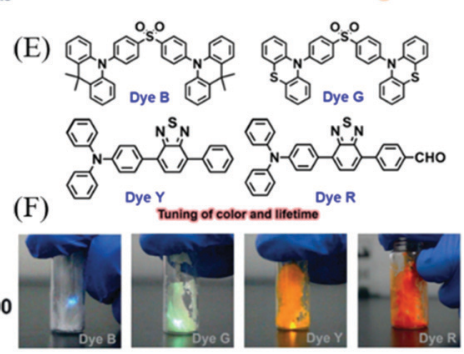

Fig. $6 \mathrm{PL}$ and ML spectra of Cz-alkyl-6 (A) and Cz-alkyl-7 (B); the inset shows the molecular structure. (C) The contactless solar-renewal process of $C z$-alkyl -6 crystals by sunlight irradiation from a solar simulator. (D) ML spectra of Cz-alkyl- 6 doping systems doped with dye B, dye $G$, dye $Y$, and dye $R$ (from left to right). (E) The molecular structure of dyes $B, G, Y$ and $R$. (F) $\mathrm{ML}$ photos of the doping system in daylight. Reproduced with permission. ${ }^{59}$ Copyright 2018, John Wiley and Sons. on the device surface, much smaller than that of typical inorganic $\mathrm{ZnS}$ (ca. $600 \mathrm{kPa})$.

\section{The ML from AIE compounds and especially the TPE derivatives}

AIE compounds usually have a flexible spatial configuration and loose molecular packing in single crystals; accordingly, they exhibited highly efficient fluorescence and mechanochromism properties in the solid-state. ${ }^{61-67}$ Once AIEgens possess ML properties, the corresponding ML spectra are similar to their PL spectra, and the ML is always so bright that it can be seen under daylight. Prior to 2015, the Tang group discovered ML from AIE compounds of complexes of $\mathrm{Cu}(\mathrm{I}) \mathrm{Cl}$, $\mathrm{Cu}(\mathrm{I}) \mathrm{Br}$, and $\mathrm{Cu}(\mathrm{I}) \mathrm{I}$ (Fig. 7A), ${ }^{60}$ which were non-emissive in solution but emitted strongly when aggregated in the crystal state with high quantum yields of $61.1-77.9 \%$ (Fig. 7B). Intense ML was observed under mechanical stimuli (Fig. 7C).

A lot of pure organic AIE compounds were reported to show bright ML, mainly from the Chi and Li groups. In 2015, Chi et al. reported the first ML from purely organic AIEgen of SPFC ${ }^{68}$ which showed a high PL $\left(\lambda_{\mathrm{em}}=518 \mathrm{~nm}\right)$ quantum yield of $93.3 \%$ and TADF properties in the solid-state. The bright green ML could be observed even under daylight, and the ML spectrum corresponded well to its PL spectrum (Fig. 8A). The dissymmetric molecular configuration induced the polar structure, and the non-centrosymmetric space group of $P n a 2_{1}$ should account for its ML property. In 2017, Tang et al. reported another compound of DBT-BZ-DMAC with the properties of TADF, AIE and ML. ${ }^{69}$ As shown in Fig. 8B, the very brilliant bluish-green 


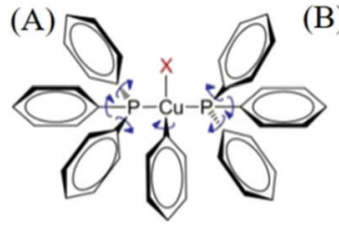

$\mathrm{Cu}(\mathrm{I}) \mathrm{X}: \mathrm{X}=\mathrm{Cl}, \mathrm{Br}$, I

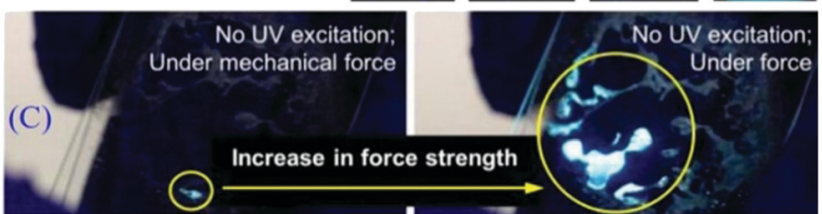

Fig. 7 (A) Chemical structures of complexes of $\mathrm{Cu}(1) \mathrm{Cl}, \mathrm{Cu}() \mathrm{Br}$, and $\mathrm{Cu}(1) \mathrm{l}$ (B) Fluorescent photographs of $\mathrm{Cu}(1) \mathrm{Cl}, \mathrm{Cu}(1) \mathrm{Br}, \mathrm{Cu}(1) !$ in solution and $\mathrm{Cu}(1) \mid$ in a pyridine/water mixture with a water volume fraction of $90 \%$. (C) The $\mathrm{ML}$ photograph of $\mathrm{Cu}(\mathrm{l}) \mathrm{I}$ under the application of increasing mechanical force without UV excitation. Reproduced with permission. ${ }^{60}$ Copyright 2015, American Chemical Society.

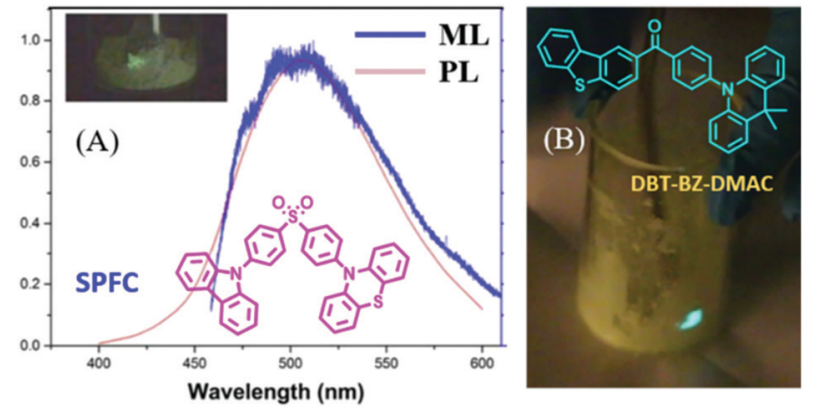

Fig. 8 (A) The PL and ML spectra of SPFC; the inset shows the molecular structure and ML photo. Reproduced with permission. ${ }^{68}$ Copyright 2015 , John Wiley and Sons. (B) The molecular structure and ML photos of DBTBZ-DMAC. Reproduced with permission. ${ }^{69}$ Copyright 2017, John Wiley and Sons.

ML was observed by scratching the crystalline powders obtained from vacuum sublimation. The single crystals of DBT-BZ-DMAC belonged to the polar space group of Pca2(1) in the orthorhombic system.

Tetraphenylethene (TPE) is one of the most famous AIEgens, and has been utilized to construct many TPE-derivatives as AIEgens, for facile syntheses, easy chemical modification, good thermal stability, and high PL quantum yields. ${ }^{60,70-74}$ Almost all of the TPE derivatives exhibited mechanochromism for the twisted molecular configuration and the loose molecular packing in crystals. However, the intermolecular interactions of TPE derivatives could be intensified by the introduction of functional groups, while the molecular polarity and crystal space groups would change accordingly. To date, lots of TPE derivatives have been reported to possess ML properties, always showing intense brightness that can be seen even under daylight, thanks to the AIE characteristics.

In 2016, Chi et al. reported bright ML from a series of TPE-based aldehydes (Fig. 9A). ${ }^{75,76}$ All of these compounds possessed AIE properties with high fluorescence quantum yields.

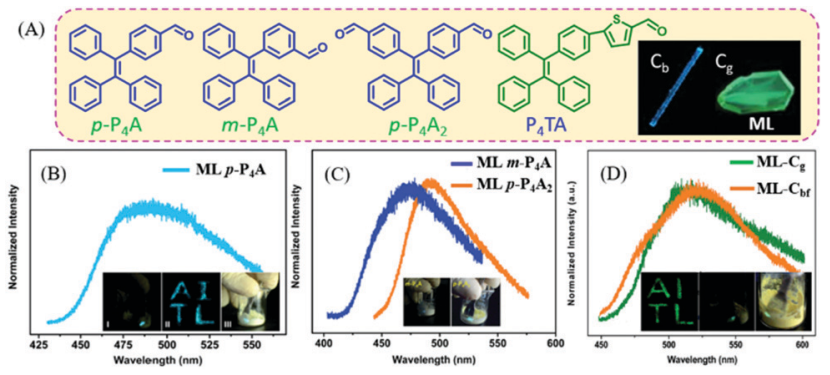

Fig. 9 (A) The molecular structures of $p-P_{4} A, m-P_{4} A, p-P_{4} A_{2}$ and $P_{4} T A$; the inset shows the $C_{b}$ and $C_{g}$ phase of $P_{4} T A$. The $M L$ spectra of $p-P_{4} A(B)$, $m-\mathrm{P}_{4} \mathrm{~A}$ and $p-\mathrm{P}_{4} \mathrm{~A}_{2}(C)$; the inset shows the $M L$ photos. Reproduced with permission. ${ }^{75}$ Copyright 2016, Royal Society of Chemistry. (D) The ML spectra of $\mathrm{P}_{4}$ TA in the $\mathrm{C}_{\mathrm{g}}$ phase and $\mathrm{C}_{\mathrm{bf}}$ phase (fumed by dichloromethane); the inset shows the ML photos. Reproduced with permission. ${ }^{76}$ Copyright 2015 , Royal Society of Chemistry.

The single crystals of $p-\mathrm{P}_{4} \mathrm{~A}$ belonged to the non-centrosymmetric polar space group of $P(2) 1$, with the presence of compact intermolecular hydrogen bonding of $\mathrm{C}-\mathrm{H} \cdots \pi$. The $\mathrm{ML}$ spectrum $\left(\lambda_{\mathrm{em}}=487 \mathrm{~nm}\right)$ of $p-\mathrm{P}_{4} \mathrm{~A}$ (Fig. 9B) corresponded well to its $\mathrm{PL}$ spectrum in the solid state, indicating the same excited state. However, when the aldehyde group was replaced by the acetyl group, or the TPE moiety with fluorene, the ML disappeared, confirming the important role of the TPE core and strong polar properties of the aldehyde group. When changing the substituent position of the aldehyde group from para- to meta-, or increasing the substituent number, the resultant compounds of $m-\mathrm{P}_{4} \mathrm{~A}$ and $p-\mathrm{P}_{4} \mathrm{~A}_{2}$ retained the $\mathrm{ML}$ character, with the maximum emission peak moving from 472 to $492 \mathrm{~nm}$ (Fig. 9C). The single crystal of $m-\mathrm{P}_{4} \mathrm{~A}$ and $p-\mathrm{P}_{4} \mathrm{~A}_{2}$ both belonged to the non-centrosymmetric polar space groups of $P(2) 1$ and $P n a(2) 1$, respectively. When the aldehyde-substituted thiophene was introduced into TPE, the $\mathrm{P}_{4}$ TA compound exhibited more interesting $\mathrm{ML}$ in two polymorphs but the same non-centrosymmetric polar space group of $P(2) 1$. The block-like polymorph $\left(\mathrm{C}_{\mathrm{b}}\right.$ phase) showed blue PL $\left(\lambda_{\mathrm{em}}=476 \mathrm{~nm}\right)$, and that of the prism-like polymorph $\left(\mathrm{C}_{\mathrm{g}}\right.$ phase $)$ was green $\left(\lambda_{\mathrm{em}}=498 \mathrm{~nm}\right)$ (Fig. 9A). However, only crystals in the $\mathrm{C}_{\mathrm{g}}$ phase exhibited bright green $\mathrm{ML}\left(\lambda_{\mathrm{em}}=517 \mathrm{~nm}\right)$ (Fig. 9D), while the crystals in the $\mathrm{C}_{\mathrm{b}}$ phase were $\mathrm{ML}$ inactive. The phase transition was observed between two crystals, and the $\mathrm{C}_{\mathrm{b}}$ phase could be induced to be ML active under fuming dichloromethane (denoted as $\mathrm{C}_{\mathrm{bf}}$ phase).

In 2016, Li et al. also reported the ML of polymorphs from TMPE (Fig. 10A). ${ }^{77}$ It was found that the block-like crystal $\left(\mathrm{C}_{\mathrm{p}}\right.$-form) was in the polar space group of $P 2_{1}(c)$, and the prism-like crystal $\left(\mathrm{C}_{\mathrm{c}}\right.$-form) in the non-polar space group of C2. The $\mathrm{C}_{\mathrm{p}}$-form and the $\mathrm{C}_{\mathrm{c}}$-form $\mathrm{PL}$ emissions peaked at 420 and $429 \mathrm{~nm}$ (Fig. 10B), respectively. However, only the $\mathrm{C}_{\mathrm{p}}$-form crystal exhibited bright deep-blue ML with a peak at $460 \mathrm{~nm}$ (Fig. 10C), while the $\mathrm{C}_{\mathrm{c}}$-form was ML inactive. What caused the totally different $\mathrm{ML}$ in two polymorphs? Although the piezoelectric effect from the polar space group could contribute to the ML, an explanation on a molecular level would be more convincing. Crystal analysis revealed that the stronger intermolecular interactions, such as $\pi-\pi$ and hydrogen bonding 

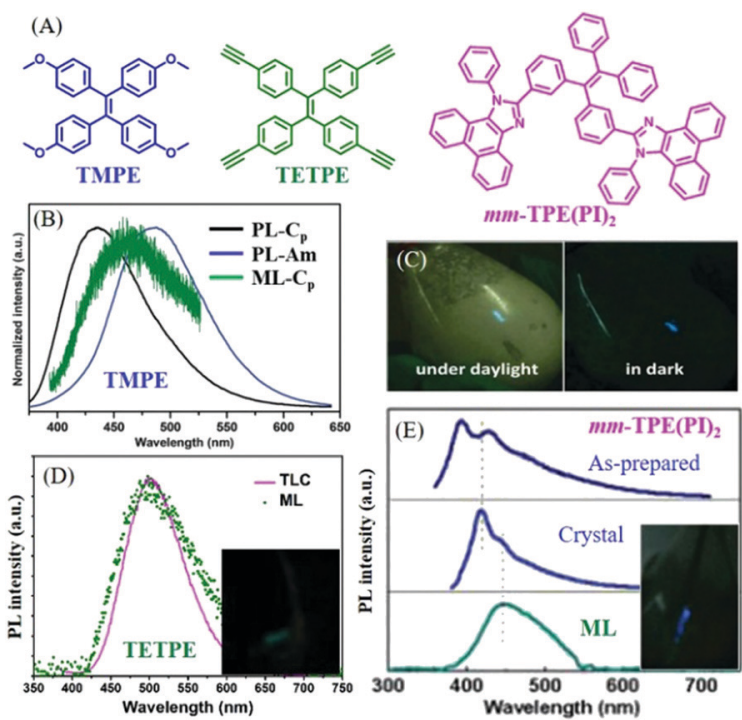

Fig. 10 (A) The molecular structures of TMPE, TETPE, and $m m-T P E(P I)_{2}$ (B) The PL spectra of TMPE in $C_{p}$ crystal form and amorphous, and the ML spectrum of $C_{p}$ crystal form. (C) ML photos of TMPE under daylight and dark conditions. Reproduced with permission. ${ }^{77}$ Copyright 2016, Royal Society of Chemistry. (D) The PL spectrum of TETPE absorbed by a TLC plate, and the ML spectrum; the inset shows a photograph of the ML. Reproduced with permission. ${ }^{78}$ Copyright 2017, Royal Society of Chemistry. (E) The PL spectra of solid $m m$-TPE $(\mathrm{PI})_{2}$ in the as-prepared state, crystal state and the ML spectrum; the inset presents the ML photo. Reproduced with permission. ${ }^{79}$ Copyright 2018, Royal Society of Chemistry.

interactions of $\mathrm{C}-\mathrm{H} \cdots \pi$ and $\mathrm{C}-\mathrm{H} \cdots \mathrm{O}$, were found in the $\mathrm{C}_{\mathrm{p}}$-form crystal, resulting in greater compression resistance. Therefore, the well-ordered crystalline phase of the $\mathrm{C}_{\mathrm{c}}$-form crystal could be easily ground to amorphous powder under a tiny force as compared to the $\mathrm{C}_{\mathrm{p}}$-form crystal. The mechanical energy might be exhausted in non-radiative pathways through the slippage of molecular layers in the $\mathrm{C}_{\mathrm{c}}$-form crystal, whereas the non-radiative transition of the $\mathrm{C}_{\mathrm{p}}$-form crystal was greatly suppressed as the intermolecular slippage was largely blocked by the rigid crystal structure; thus, ML was produced since the radiative channel would open under the mechanical stimuli.

In $2017, \mathrm{Li}$ et al. further reported green $\mathrm{ML}\left(\lambda_{\mathrm{em}}=498 \mathrm{~nm}\right)$ from a pure hydrocarbon TPE derivative of TETPE (Fig. 10A and D), ${ }^{78}$ and the ML spectrum corresponded well with the PL spectrum of TETPE absorbed on a thin-layer chromatography (TLC) plate. It was interesting to find that TETPE was nonpolar and belonged to the centrosymmetric space group $C 2$ (indicating the non-piezoelectric property), which was different from previous ML compounds. TETPE crystal emitted blue-greenish light peaked at $451 \mathrm{~nm}$, and possessed strong intermolecular interactions. Since the crystal was difficult to grind into fine amorphous powder, TETPE showed weak mechanochromism properties. The triple bond of $\mathrm{C} \equiv \mathrm{C}$ can also induce strong intermolecular interactions because of the positive electronic properties of terminal alkynes, induced by the electron-withdrawing $\mathrm{C}$ atom in the sp hybridization. ML could be ascribed to the strong static electronic interactions.
In 2018, also from the Li group, another deep blue ML from a TPE derivative of $m m$-TPE(PI $)_{2}$ was reported. ${ }^{79}$ As shown in Fig. 10A, two phenanthro[9,10- $d]$ imidazole groups were introduced to the meta-positions of TPE. The ML emission maximum was observed at $445 \mathrm{~nm}$, which red-shifted to the corresponding PL $\left(\lambda_{\mathrm{em}}=417 \mathrm{~nm}\right)$ in the crystal state (Fig. 10E). The $m m$-TPE(PI $)_{2}$ also exhibited the centrosymmetric space group of $P 2_{1} / n$, and the ML could be ascribed to the large molecular dipole moment and very tight molecular packing in the crystal.

In 2017, Thilagar et al. reported ML from a diarylborylphenothiazine derivative of DMPBPTZ ${ }^{80}$ (Fig. 11A), which could be regarded as a TPE analog due to the isoelectronic property of the $\mathrm{B}-\mathrm{N}$ bond as compared to the $\mathrm{C}=\mathrm{C}$ one. Really, DMPBPTZ was AIE active with the mechanochromism property. The crystal belonged to the polar space group of $R 3 c$, and emitted green light with a peak at $513 \mathrm{~nm}$ (Fig. 11B). The bright greenish-yellow $\mathrm{ML}\left(\lambda_{\mathrm{em}}=530 \mathrm{~nm}\right.$ ) was observed (which red-shifted) under mechanical stimuli rather than PL. Ghosh et al. further connected the diarylboryl group to the phenyl unit of phenothiazine, and the resultant compound of BMBPTZ (Fig. 11A) also exhibited green ML. ${ }^{81}$ Because of the polar electron donor-acceptor molecular structure, the PL of BMBPTZ redshifted from 491 to $520 \mathrm{~nm}$ after grinding, demonstrating mechanochromism (Fig. 11C). The ML spectrum $\left(\lambda_{\mathrm{em}}=520 \mathrm{~nm}\right)$ corresponds well to the PL spectrum of the ground sample. As expected, the single crystal of BMBPTZ belonged to the non-centrosymmetric space group of $P 31$.

\section{Molecular configuration-related ML luminogens}

As mentioned above, the ML spectra displayed emission characteristics of the corresponding PL spectra. Although some compounds exhibited changeable PL spectra in the solid-state as a result of mechanochromism, most of the ML spectra resembled
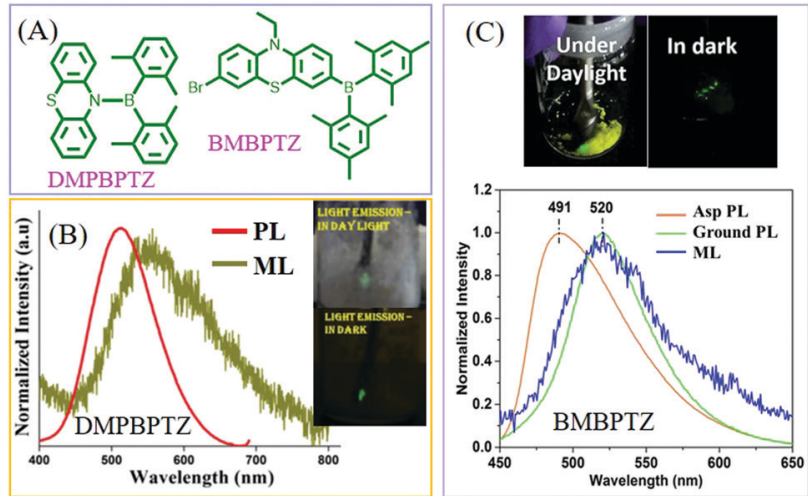

Fig. 11 (A) The molecular structures of DMPBPTZ, and BMBPTZ. (B) The $\mathrm{PL}$ and ML spectra of DMPBPTZ. Reproduced with permission. ${ }^{80}$ Copyright 2017, Royal Society of Chemistry. (C) The PL spectra of the as-prepared (Asp) and ground sample, and the ML spectrum of BMBPTZ. The inset shows the ML photos under daylight and dark conditions. Reproduced with permission. ${ }^{81}$ Copyright 2017, John Wiley and Sons. 
PL in the single molecular state, probably because ML was produced at the cracked surface of the crystals. However, the PL of some compounds was strongly correlated with molecular configurations, and the PL characteristics were retained in the crystals. Therefore, the ML spectra should display these characters as well.

In 2018, Li et al. reported interesting ML phenomena: the pyrene derivatives of Py-Bpin and $\mathrm{Py}-\mathrm{Br}$ (Fig. 12A) had $\mathrm{ML}$ spectra composed of both monomer and excimer emissions. ${ }^{82}$ As shown in Fig. 12B, in the THF/water mixture solution of Py-Bpin, the PL intensity gradually increased when the water fracture $\left(f_{\mathrm{W}}\right)$ increased from 0 to $80 \%$. However, a distinct new red-shifted emissive band appeared at around $460 \mathrm{~nm}$ and further increased at higher $f_{\mathrm{W}}$, which should be ascribed to the excimer emission since the pyrene easily formed a dimer because of the planar structure. Both $\mathrm{Py}-\mathrm{Br}$ (Fig. 12C) and Py-Bpin (Fig. 12D) can emit bright ML that can be observed under daylight. However, only one emission band was observed at around $459 \mathrm{~nm}$ in the ML spectrum of $\mathrm{Py}-\mathrm{Br}$, which corresponds well to the excimer emission in the solid-state. In contrast, Py-Bpin showed a much different ML spectrum than that of PL. The emission bands around 425 and $450 \mathrm{~nm}$ corresponded well to the single molecular emission, while the red-shifted emission band around $470 \mathrm{~nm}$ was consistent with the excimer emission in the solid-state. The excimer emission of ML indicated that the molecules inherited the luminous characteristics in the aggregated state, and the intermolecular interactions and molecular aggregates also contributed significantly to ML.

Also in 2018, the $\mathrm{Li}$ group reported a changeable $\mathrm{ML}$ phenomenon in the phenothiazine derivative of FCO-CzS (Fig. 13A), ${ }^{83}$ whose ML color changed from blue to white and
(A)

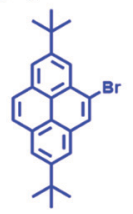

$\mathrm{Py}-\mathrm{Br}$

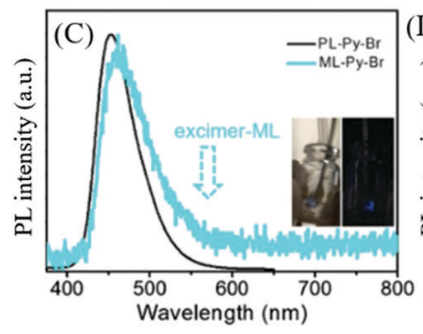

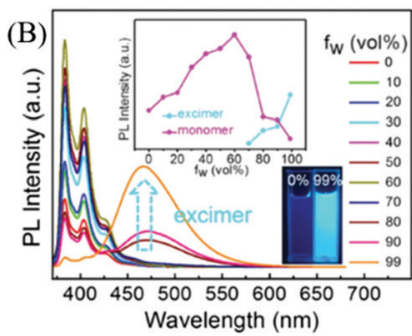

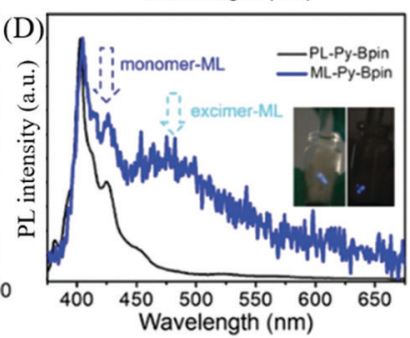

Fig. 12 (A) The molecular structures of Py-Br and Py-Bpin. (B) PL spectra and the intensity of the monomer and excimer of Py-Bpin in THF/water mixtures with different water fractions; the inset shows photos of Py-Bpin in the mixture solution with water fractions of 0 and $99 \%$, under the irradiation of $365 \mathrm{~nm}$ UV light. PL and ML spectra of (C) Py-Br (D) and Py-Bpin; the inset shows the ML photos under daylight and in dark conditions. Reproduced with permission. ${ }^{82}$ Copyright 2018 , John Wiley and Sons. yellow upon continuous mechanical stimuli. As shown in Fig. 13D, the dynamic ML spectra upon grinding were recorded. From Fig. 13B, the FCO-CzS crystal exhibited blue ML at the initial stage of grinding, with only one main emission band present at around $449 \mathrm{~nm}$ in the ML spectrum. However, the ML color changed to white upon further grinding, and another emission band arose at around $570 \mathrm{~nm}$ and gradually increased in accordance with the fracture of the crystal. The white emission was composed of blue and yellow ML emissions. As the crystal was ground into fine powder, the ML turned to yellow and only one emission band was seen at around $570 \mathrm{~nm}$ in the ML spectrum. The CIE coordinates changed from $(0.27,0.30)$ (blue) to $(0.30,0.35)$ (white), then to $(0.43,0.47)$ (yellow). The PL analysis revealed that the FCO-CzS was AIE active (Fig. 13A), and exhibited mechanochromism (Fig. 13E) similar to the ML changes. The crystal emitted fluorescence ( $\lambda_{\mathrm{em}}=434 \mathrm{~nm}, \tau=0.5 \mathrm{~ns}$ ) with a quantum yield of $2 \%$. The PL color changed to white and yellow upon continuous grinding, with the quantum yield increasing to $11 \%$; the yellow emission peak at $544 \mathrm{~nm}$ appeared to be TADF with a lifetime of $5.1 \mathrm{~ms}$. The novel PL and ML changes upon grinding could not be ascribed to the changes in the molecular packing from the crystal to the amorphous phase, but the molecular configurations should be considered. There are two configurations of phenothiazine, namely, quasi-axial and quasi-equatorial; the former has a higher energy gap and the latter has better conjugation structure. Therefore, the dynamic PL and ML should be explained by the molecular conformation transition of phenothiazine from quasi-axial to quasi-equatorial upon mechanical stimuli (Fig. 13C). The crystal structure also revealed that FCO-CzS was quasi-axial, which was also consistent with the phenomenon that the PL efficiency was low in the crystals but much higher after grinding.

In 2018, Chi et al. also reported a color-changeable ML from CDpP (Fig. 14A). ${ }^{84}$ Two kinds of crystals CDpP-B and CDpP-G were cultured in the space groups of $P 2_{1} / n$ and $C c$, respectively. As shown in Fig. 14B, the CDpP-B crystal emitted blue ML
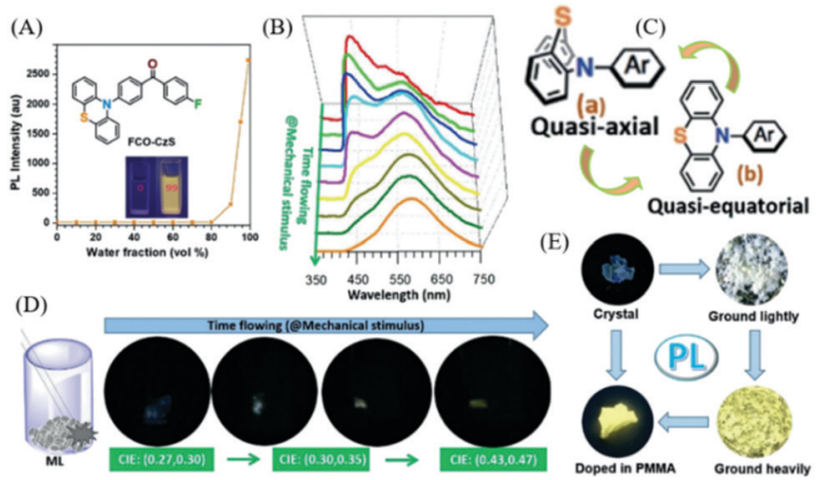

Fig. 13 (A) PL intensity of FCO-CzS in a mixed solution of THF/water with different water fractions; the inset shows the photos of $\mathrm{FCO}-\mathrm{CzS}$ in mixed solution with water fractions of 0 and $99 \%$, under UV irradiation at $365 \mathrm{~nm}$. (B) The ML spectra with different grinding times. (C) Quasi-axial and quasiequatorial molecular configurations of phenothiazine. (D) ML photographs of $\mathrm{FCO}-\mathrm{CzS}$ with different grinding times. (E) Photographs of FCO-CzS in different solid states taken under UV irradiation at $365 \mathrm{~nm}$. Reproduced with permission. ${ }^{83}$ Copyright 2018, John Wiley and Sons. 

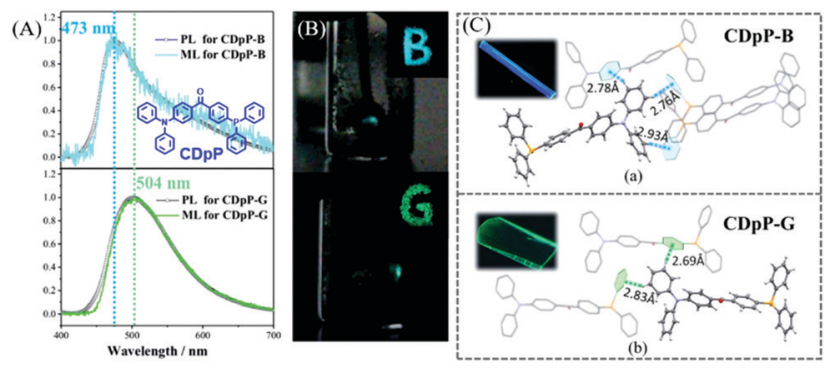

Fig. 14 (A) PL and ML spectra of CDpP-B and CDpP-G; the inset shows the molecular structure of CDpP. (B) Photos of the solid-state PL with letters of " $B$ " and " $G$ " under the irradiation of $365 \mathrm{~nm}$ UV light; ML photos of CDpP-B (above) and CDpP-G (below). (C) Single crystal structures of $\mathrm{CDpP}-\mathrm{B}$ and $\mathrm{CDpP}-\mathrm{G}$ with intermolecular $\mathrm{C}-\mathrm{H} \cdots \pi$ interactions; the inset shows the corresponding luminescence photos under the irradiation of 365 nm UV light. Reproduced with permission. ${ }^{84}$ Copyright 2018, Royal Society of Chemistry.

$\left(\lambda_{\mathrm{em}}=473 \mathrm{~nm}\right)$ while the green ML for the CDpP-G crystal $\left(\lambda_{\mathrm{em}}=504 \mathrm{~nm}\right)$, corresponded well to their PL spectra. The molecular geometry difference between two crystals is quite small, however, the intermolecular interactions of hydrogen bonding were different because of the different molecular packing in the crystals (Fig. 14C). Combined with the femtosecond transient spectra, the tunable ML of $\mathrm{CDpP}$ mainly originated from the different $\mathrm{C}-\mathrm{H} \cdots \pi$ interactions in the crystal. Crystalline CDpP-B exhibited more $\mathrm{C}-\mathrm{H} \cdots \pi$ interactions, and the blue ML could be ascribed to the locally excited (LE)-state since the twisting process in the excited state was restricted. However, the $\mathrm{C}-\mathrm{H} \cdots \pi$ interactions in the $\mathrm{CDpP}-\mathrm{G}$ crystals were much fewer, and the green ML arose from the twisted intramolecular charge transfer (TICT) excited state as the diphenylamine moiety was relatively free to rotate. In this case, the weak intermolecular interactions in the crystal could also be used to tune the color of the ML.

\section{The ML of compounds consisting of the dual emission of fluorescence and phosphorescence}

Generally, the phosphorescence of purely organic compounds is hard to observe at room temperature because of the spinforbidden transition between the ground state and the triplet excited state. However, recent research has revealed that strong and persistent room-temperature phosphorescence (RTP) can be observed in molecular aggregates. ${ }^{85-88}$ The abnormal RTP was considered to originate from intermolecular interactions since the triplet exciton could be converted from a singlet activated by the spin-orbital coupling effect, and followed by stabilization through the delocalization of intermolecular orbitals. In 2018, the Li group reported the first example of photoinduced phosphorescence in $\mathrm{CS}_{-} \mathrm{CF}_{3}{ }^{89} \mathrm{CS}^{-\mathrm{CF}_{3}}$ did not exhibit visible RTP in the as-prepared single crystal sample; however, strong RTP with a lifetime of $299 \mathrm{~ms}$ was observed after irradiation by a normal UV lamp for $5 \mathrm{~min}$, which returned to the initial state after two hours of standing under ambient conditions. The crystal structure analysis revealed that the intermolecular distance was shorter after UV irradiation, which led to stronger $\pi-\pi$ interactions. The mechanical source is probably a more powerful excitation source than the photo source, and the mechano-phosphorescence was previously reported in some organic crystals. More excitingly, mechanically induced RTP and photosensitive switching between RTP and ML were recently discovered, indicating the peculiar properties of solid-state luminescence. Overall, although there have been some reports of mechanophosphorescence produced by non-phosphorescent crystals at room temperature, ${ }^{43,90}$ the mechanophosphorescence was often inherited from solid-state phosphorescence properties at room temperature, and the ML spectra were composed of both fluorescence and phosphorescence. As the photo-sensitive crystals underwent structural changes under photoirradiation, the ML properties changed accordingly.

In 2017, Li et al. reported dual ML comprised of fluorescence $\left(\lambda_{\mathrm{em}}=350 \mathrm{~nm}\right)$ and phosphorescence $\left(\lambda_{\mathrm{em}}=450 \mathrm{~nm}\right)$ in DPP-BO (Fig. 15). ${ }^{91}$ However, the solid DPP-BO could not emit phosphorescence under ambient conditions, according to the PL spectrum, indicating that the excited state produced from mechanical stimuli should be different from that of photoirradiation.

The DPP-BO crystal belongs to the non-centrosymmetric Cmc21 space group. The efficient intermolecular and intramolecular interactions in the crystal enhance the intersystem crossing from the singlet to the triplet state, since the electronic excitation caused by the mechanical stimuli might be a much stronger excitation source than the photostimuli.

In 2017, Li et al. reported compounds of $\mathrm{CzS}^{-\mathrm{CH}_{3}}$ and $\mathrm{CzS}_{-} \mathrm{C}_{2} \mathrm{H}_{5}$ that possessed both RTP and ML properties. ${ }^{92}$ At room temperature, the PL of the $\mathrm{CzS}_{-} \mathrm{C}_{2} \mathrm{H}_{5}$ crystal was composed of fluorescence $\left(\lambda_{\mathrm{em}}=426\right)$ and RTP $\left(\lambda_{\mathrm{em}}=497, \tau=4.59 \mathrm{~ms}\right)$, and bright blue ML was observed with scraping (Fig. 16A); there was one main emission peak at around $430 \mathrm{~nm}$ and another peak tailing in the long wavelength region, which exhibited both fluorescence and phosphorescence. After thorough grinding, the high-contrast mechanochromism effect was observed in the CzS- $\mathrm{C}_{2} \mathrm{H}_{5}$ crystal with the color of PL changing from green to blue, and the resultant PL spectrum had similar characteristics to the
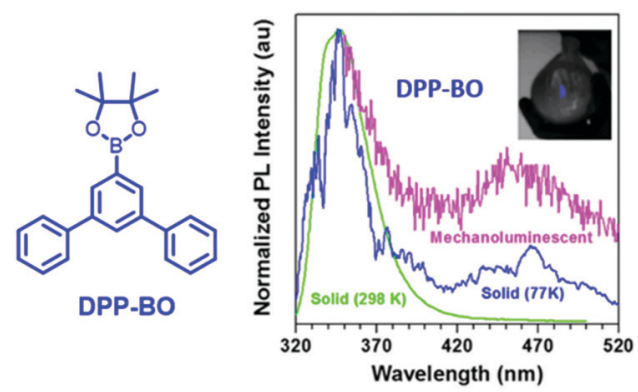

Fig. 15 The molecular structure of DPP-BP; the PL spectra of solid DPP-BO at $298 \mathrm{~K}$ and $77 \mathrm{~K}$, and the mechanoluminescent (ML) spectrum. Inset: The ML of the as-prepared DPP-BO sample upon grinding with a spatula in daylight. Reproduced with permission. ${ }^{92}$ Copyright 2017 , John Wiley and Sons. 

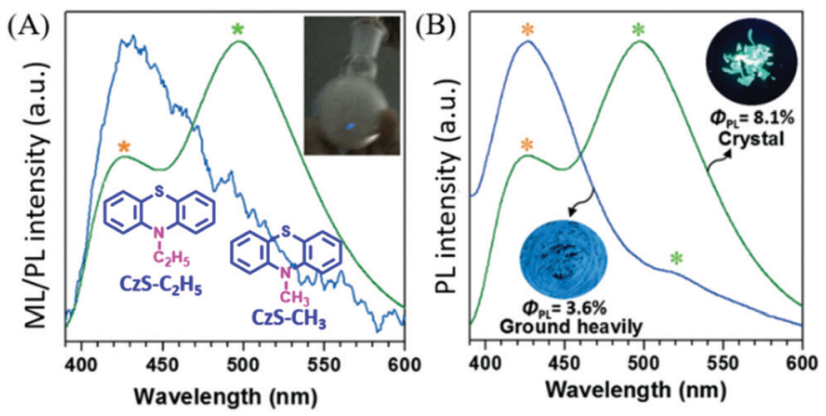

Fig. 16 (A) The $\mathrm{ML}$ and $\mathrm{PL}$ spectra of $\mathrm{CzS}-\mathrm{C}_{2} \mathrm{H}_{5}$ crystals. Inset: The molecular structure of $\mathrm{CzS}-\mathrm{C}_{2} \mathrm{H}_{5}, \mathrm{CzS}-\mathrm{CH}_{3}$, and the $\mathrm{ML}$ image of the $\mathrm{CzS}$ $\mathrm{C}_{2} \mathrm{H}_{5}$ sample upon grinding with a glass rod in daylight. (B) The prompt PL spectra and photos of crystals of $\mathrm{CzS}-\mathrm{C}_{2} \mathrm{H}_{5}$ and after grinding heavily, under 365 nm UV-irradiation. Reproduced with permission. ${ }^{91}$ Copyright 2017, John Wiley and Sons.

ML spectrum. As a result, the corresponding RTP lifetime decreased to $1.59 \mathrm{~ms}$, and the PL quantum yield decreased from $8.1 \%$ to $3.6 \%$ (Fig. 16B). Similar emission behaviour was also observed in $\mathrm{CzS}-\mathrm{CH}_{3}$ for the RTP and mechanochromism properties; however, the ML was much weaker and could only be observed in the dark due to the low PL efficiency $\left(\Phi_{\mathrm{PL}}=3.0 \%\right.$ in crystal, $2.1 \%$ after grinding).

In 2018, Babu et al. also reported a phosphorescence-type ML in PT-Bpin (Fig. 17C) at room temperature. ${ }^{93}$ PT-Bpin had mechanochromism, with its emission changing from blue to yellow upon grinding (Fig. 17A); accordingly, the RTP emission at around $590 \mathrm{~nm}(\tau=34 \mathrm{~ms})$ gradually disappeared. At $77 \mathrm{~K}$, the frozen solution of PT-Bpin in 2-methyltetrahydrofuran emitted a golden yellow phosphorescence with a lifetime of 1.7 s. Because of the similarity of the ML spectrum to the phosphorescence spectrum at $77 \mathrm{~K}$ (Fig. 17D), the ML should be phosphorescence, and the ground sample gradually lost ML in air (Fig. 17B). The single-crystal analysis revealed that the sterically bulky groups around PT-BPin prevented the compact molecular packing that could relieve the quenching of triplet excitons.

In 2018, Li et al. also reported distinct ML from the BrFlu-CBr crystal (Fig. 18E), ${ }^{94}$ in which, the bromine atoms were introduced to increase the spin-orbital coupling and intersystem crossing, and strong $\mathrm{Br} \cdots \mathrm{Br}$ interactions were found in the crystal. BrFlu-CBr exhibited RTP emission at $480 \mathrm{~nm}$ upon UV irradiation (Fig. 18A), and the phosphorescence efficiency was $4.56 \%$. More interestingly, as shown in Fig. 18B, after mechanical stimuli, cyan ML was observed, which consisted of the dual emission of fluorescence and phosphorescence, similar to the corresponding PL spectra. Further investigation revealed that after the mechanical stimuli, the green-white ML could still be observed under soft light (Fig. 18C). The ML spectrum was consistent well with the phosphorescence spectrum of the BrFlu-CBr crystal, confirming the mechanically induced RTP. After continuous grinding, the weak blue ML was observed from the obtained BrFlu-CBr powder (Fig. 21D), and the emission band in the long-wavelength region decreased in the ML spectrum. Therefore, the tri-color ML was obtained,
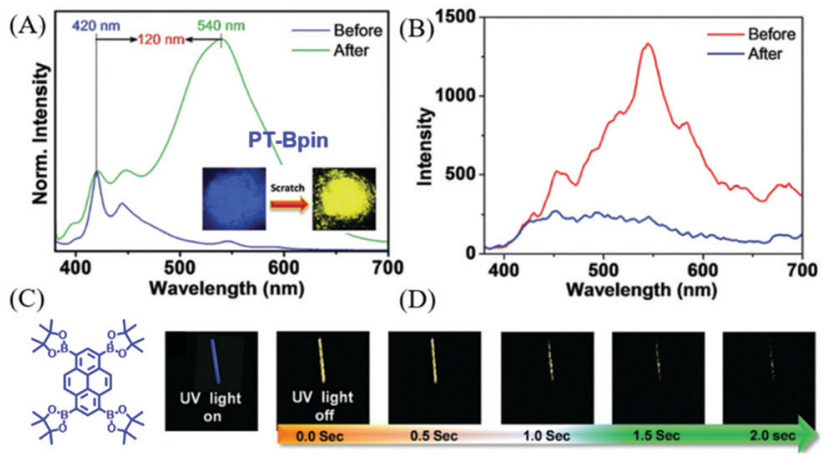

Fig. 17 (A) The PL spectra of PT-Bpin before and after grinding/scratching for 2 min under the UV irradiation of $365 \mathrm{~nm}$. The inset shows the corresponding photos. (B) Phosphorescence spectra of the solid at RT before and after grinding. (C) The molecular structure of PT-Bpin. (D) Phosphorescence images of the PT-Bpin glassy MTHF solution (at $77 \mathrm{~K}$ for $1 \mathrm{~min}$ ) in a quartz tube $(3 \mathrm{~mm}$ ) recorded at different time intervals upon turning off the excitation source $(365 \mathrm{~nm})$ after $3 \mathrm{~s}$ of exposure. Reproduced with permission. ${ }^{93}$ Copyright 2018, Royal Society of Chemistry.
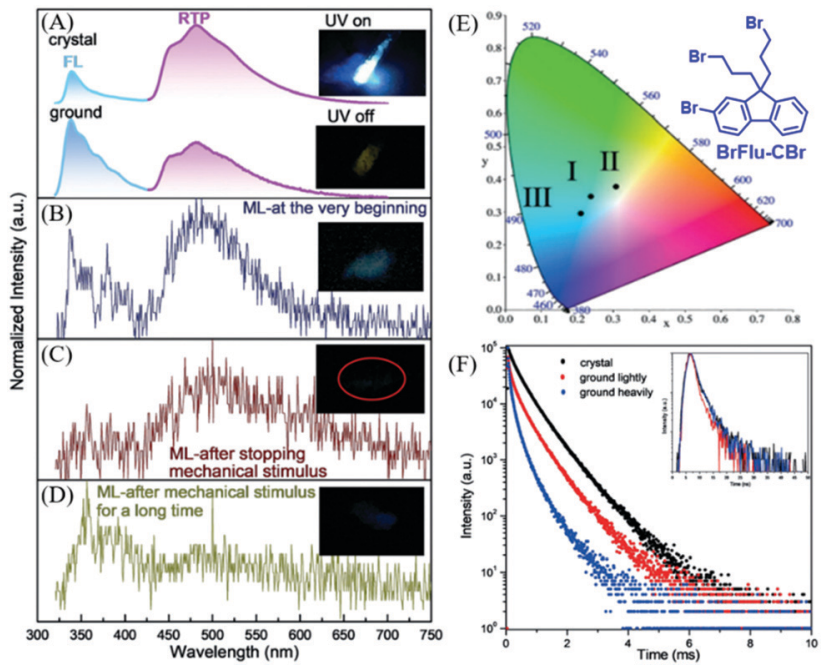

Fig. 18 (A) PL spectra of crystals (top) and powder (after light grinding; bottom) of BrFlu-CBr. Inset: Photos of the luminescence of crystals of BrFlu-CBr before and after UV excitation (365 nm). (B) ML spectrum of $\mathrm{BrFlu}-\mathrm{CBr}$ at the beginning; the inset shows the corresponding $\mathrm{ML}$ image. (c) The $\mathrm{ML}$ spectrum of BrFlu-CBr after removing the mechanical stimulus; the inset shows the corresponding $M L$ image. (D) The $M L$ spectrum of BrFlu-CBr after removing the mechanical stimulus after a long time; the inset shows the corresponding $\mathrm{ML}$ images. (E) The calculated $\mathrm{CIE}$ coordinates in the CIE 1931 color space chromaticity diagram based on the ML spectra at different times: I, at the very beginning; II, after stopping mechanical stimulus; III, after mechanical stimulus for a long time. (F) Phosphorescence decay curves of BrFlu- $\mathrm{CBr}$ in different states, inset: fluorescence decay curves of BrFlu- $\mathrm{CBr}$ in different states. Reproduced with permission. ${ }^{94}$ Copyright 2018, John Wiley and Sons.

and the corresponding CIE coordinates are presented in Fig. 18E. The ML under the different conditions obviously revealed that the molecular packing in the crystal played an important role in regulating the phosphorescence intensity and lifetime. The phosphorescence decay spectra also supported that the crystal state of BrFlu-CBr exhibited higher 
phosphorescence lifetime, which gradually decreased on further grinding (Fig. 18F).

Phthalimide derivatives containing a trifluoromethylphenyl group have been reported to exhibit colorful $\mathrm{ML}$, due to the dipolar molecular structure and the non-centrosymmetric space group. ${ }^{95,96}$ However, their phosphorescence properties have not been reported. In 2018, Chi et al. reported both phosphorescence and ML of phthalimide derivatives of ImF and $\operatorname{ImBr}$ (Fig. 19C). As shown in Fig. 19A, the ImF crystal could emit strong blue fluorescence $\left(\lambda_{\mathrm{em}}=455 \mathrm{~nm}, \tau=16.8 \mathrm{~ns}\right)$ under the irradiation of $365 \mathrm{~nm}$ UV light, and the RTP was also observed at around $555 \mathrm{~nm}$ with the lifetime of $35.3 \mathrm{~ms}$. When the fluorine atom was substituted by the bromine one, the resultant $\mathrm{ImBr}$ exhibited extraordinary dual-emissive bands (Fig. 19A). It was interesting to find that the blue emission peak at $457 \mathrm{~nm}$ showed a fast decay lifetime of $2.6 \mathrm{~ns}$, and an intense emission band at around $548 \mathrm{~nm}$ with an ultralong lifetime of $102.1 \mathrm{~ms}$. The RTP could be gradually intensified to the maximum in about $0.8 \mathrm{~s}$ upon excitation with UV light (Fig. 19B), and the corresponding emission color changed from blue to white (Fig. 19E). After ceasing excitation, strong yellow emission was observed for ImBr. The crystalline powder of ImF exhibited ML with the corresponding spectrum $\left(\lambda_{\mathrm{em}}=462 \mathrm{~nm}\right)$ being similar to its PL spectrum (Fig. 19F and G). The similar color of ML was also observed for $\mathrm{ImBr}$, but further investigation revealed that the
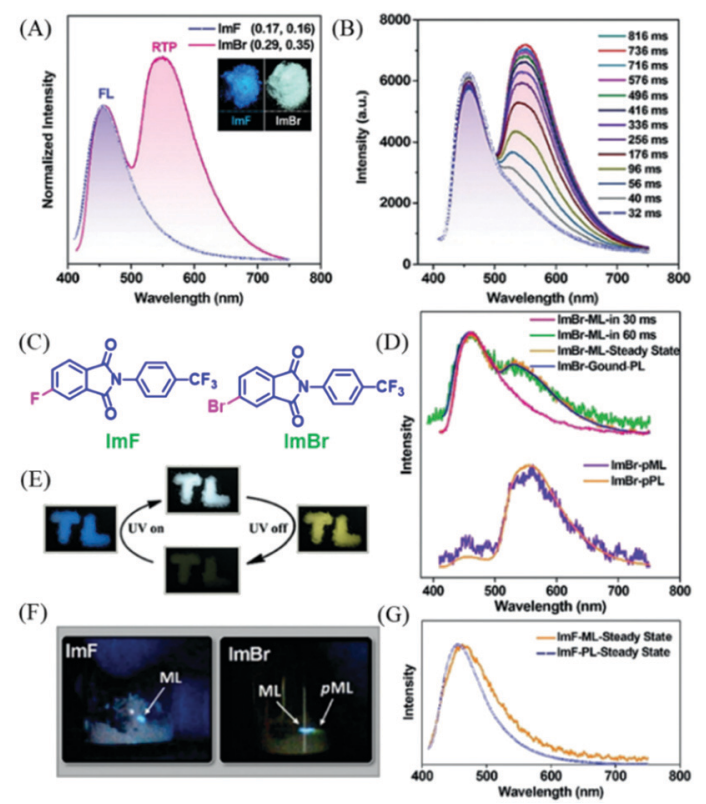

Fig. 19 (A) PL spectra of $\mathrm{ImF}$ and $\mathrm{ImBr}$ in the solid-state $\left(\lambda_{\mathrm{Ex}}=365 \mathrm{~nm}\right)$; the inset shows the luminescence photos of the crystalline powders under the illumination of $365 \mathrm{~nm}$ UV light. (B) PL spectra of the $\mathrm{ImBr}$ crystalline powder under the illumination of $365 \mathrm{~nm}$ UV light. (C) The molecular structure of $\mathrm{ImF}$ and $\mathrm{ImBr}$. (D) Transient and persistent $\mathrm{ML}$ and PL spectra of the pristine and ground samples of $\mathrm{ImBr}$. (E) Tricolor emission switching of $\mathrm{ImBr}$. The color of "TL" changed from blue to white under excitation of $365 \mathrm{~nm}$ UV light. After turning off the UV, the color changed to yellow. (F) ML and $\mathrm{pML}$ photos of $\mathrm{ImF}$ and $\mathrm{ImBr}$ taken at room temperature. (G) ML and $\mathrm{PL}$ spectra of $\mathrm{ImF}$ at room temperature. Reproduced with permission. ${ }^{97}$ Copyright 2018, John Wiley and Sons. transient $\mathrm{ML}$ changed from fluorescence $\left(\lambda_{\mathrm{em}}=460 \mathrm{~nm}\right)$ to fluorescence-phosphorescence dual emission $\left(\lambda_{\mathrm{em}}=462\right.$, $527 \mathrm{~nm}$ ) in about $60 \mathrm{~ms}$ (Fig. 19D and F). While the steadystate ML spectrum corresponded well to the PL spectrum of the ground sample, it was amazing to find that following the blue ML at the force-bearing point, the yellow afterglow ribbon lasted for about one second. Thus, ImBr was persistently $\mathrm{ML}$ ( $p$-ML) active. The spectrum of $p$-ML also showed similar emission bands to the PL (Fig. 19D), indicating that the $p$-ML experienced the same photophysical process, regardless of the different excitation mode.

In 2018, Chi et al. also found the similar $p$-ML phenomenon in compound CX (Fig. 20A). ${ }^{98}$ Under the excitation of $365 \mathrm{~nm}$ UV light, the yellow RTP (Fig. 20B) was observed in the crystal of CX49, which was recrystallized from a mixture solvent of dichloromethane and $n$-hexane $(4 / 9, v / v)$. As shown in Fig. 20C, the dual emission of fluorescence $\left(\lambda_{\mathrm{em}}=460, \tau=7.2 \mathrm{~ns}\right)$ and phosphorescence $\left(\lambda_{\mathrm{em}}=545, \tau=0.15 \mathrm{~s}\right)$ had the overall quantum yield of $40 \%$. The yellow $p$-ML was also observed after ceasing the mechanical stimuli at room temperature (delayed about $30 \mathrm{~ms}$, Fig. 20E), and $p$-ML was even brighter at $77 \mathrm{~K}$. The ML spectrum with an emission peak at $545 \mathrm{~nm}$ was similar to that of RTP (Fig. 20D).

In 2019, Lin et al. reported a carbazole derivative of 2-(9H-carbazol-9-yl)ethanol (CZEO) with both ML and photoinduced RTP properties. ${ }^{99}$ The lifetime of CZEO at $555 \mathrm{~nm}$ was $15.6 \mathrm{~ns}$, indicating the fluorescence properties. However, the lifetime increased to $739.6 \mathrm{~ms}$ after being irradiated for $2 \mathrm{~min}$ with UV light at $365 \mathrm{~nm}$ (Fig. 21A and C). The activated CZEO spontaneously deactivated when the UV irradiation stopped, and the RTP lifetime decreased to $0.03 \mathrm{~ms}$ after being deactivated for $560 \mathrm{~s}$. Therefore, the photo-activated CZEO was metastable and would turn back to the pristine state after ceasing UV irradiation for several minutes. More interestingly, the CZEO crystal exhibited obvious deep blue ML under mechanical stimuli (Fig. 21B), and the ML spectrum corresponded well to its PL spectrum. However, the
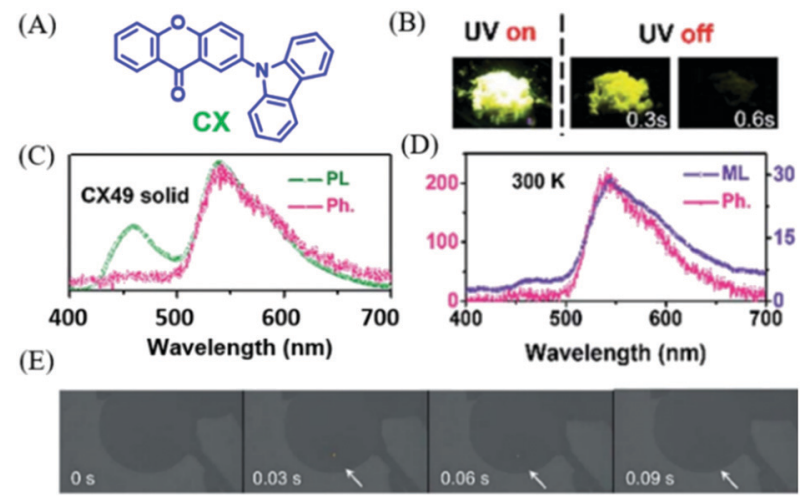

Fig. 20 (A) The molecular structure of CX. (B) Photos of CX49 crystals before and after the UV-light irradiation. (C) PL phosphorescence (Ph, delayed $8 \mathrm{~ms}$ ) spectra of $\mathrm{CX}$ in the crystalline (CX49) state. (D) Phosphorescence and ML spectra of CX49 crystals at $300 \mathrm{~K}$. (E) ML photos of the CX49 crystal at $77 \mathrm{~K}$, showing mechanically-induced persistent phosphorescence. Reproduced with permission. ${ }^{98}$ Copyright 2018, Royal Society of Chemistry. 


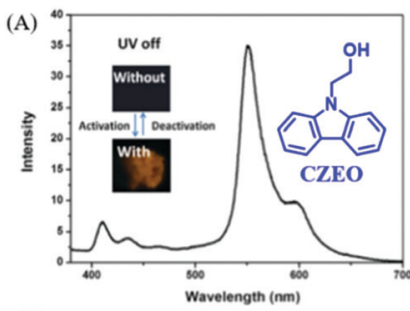

(C)

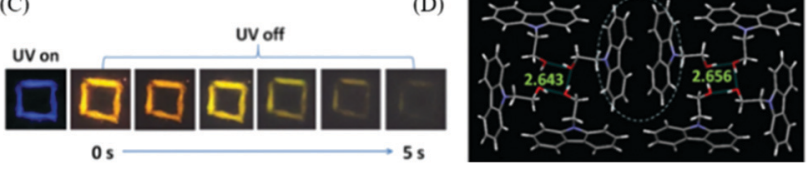

Fig. 21 (A) RTP spectrum of the photo-activated CZEO, $\lambda_{E x}=364 \mathrm{~nm}$, inset presents the photos of CZEO upon activation by UV light. (B) The ML spectrum of the photo-activated CZEO; the inset shows the ML photos of CZEO. (C) Photos of the UV-activated CZEO before and after the UV light was turned off. (D) The packing structure of CZEO in a unit cell; the $\mathrm{C}-\mathrm{H} \cdots \pi$ of hydrogen bond distance was also listed. Reproduced with permission. ${ }^{99}$ Copyright 2018, Royal Society of Chemistry.

ML activity was switched off after being activated by UV irradiation for $2 \mathrm{~min}$, demonstrating UV turn-on RTP and turn-off ML properties. The analysis of single crystals revealed that there were 16 molecules in one unit cell, and the cluster consisting of four molecules was fixed by hydrogen bonds with the $\mathrm{C}-\mathrm{H} \cdots \pi$ distance of 2.643 or $2.656 \AA$ (Fig. 21D). The crystals were found to become tighter after photo-activation. Thus, the pristine looser crystal was easily separated by external mechanical stimuli, producing an electric field for the excitation of ML. After UV activation, the ML was switched off since the tighter crystal was difficult to destroy, and the RTP was generated as the nonradiative transition rate was suppressed. It was interesting that a similar photo stimuli response of the ML and RTP phenomena was found in a previously reported molecule of NIPC, in which, the RTP lifetime $\left(\lambda_{\mathrm{em}}=550 \mathrm{~nm}, \tau=82.1 \mathrm{~ms}\right)$ was improved by about 10 times ( $833.9 \mathrm{~ms}$ ) after being activated by UV light for $2 \mathrm{~min}$, whereas the ML character disappeared.

In 2019, Li et al. reported an interesting compound of TPA-B (Fig. 22A) with the combined properties of ML and photoinduced RTP. ${ }^{100}$ The deep blue ML was observed in TPA-B upon grinding (Fig. 23D), and there were no obvious responses or changes in ML during UV irradiation. Nevertheless, although no RTP was observed at the initial stage of UV irradiation, the intense yellow afterglow was observed and lasted for $1.0 \mathrm{~s}$ after the 20 min irradiation (Fig. 22B and C), and the corresponding RTP lifetime increased from 5.32 to $211.13 \mathrm{~ms}$. Meanwhile, the fluorescence at $410 \mathrm{~nm}$ was enhanced by about two times with the lifetime increasing from 1.29 to $1.48 \mathrm{~ns}$. After ceasing the UV irradiation, the photoinduced RTP lifetime and intensity decreased, and finally went to the original state after storage for over $100 \mathrm{~min}$. At $77 \mathrm{~K}$, the $\mathrm{ML}$ was similar to PL, and the phosphorescence peak at $525 \mathrm{~nm}$ could be observed in the ML spectrum. However, the phosphorescence demonstrated little change upon UV irradiation at $77 \mathrm{~K}$, indicating that the photoinduced RTP should be ascribed to the molecular motion in crystals at room temperature, as molecular motion was
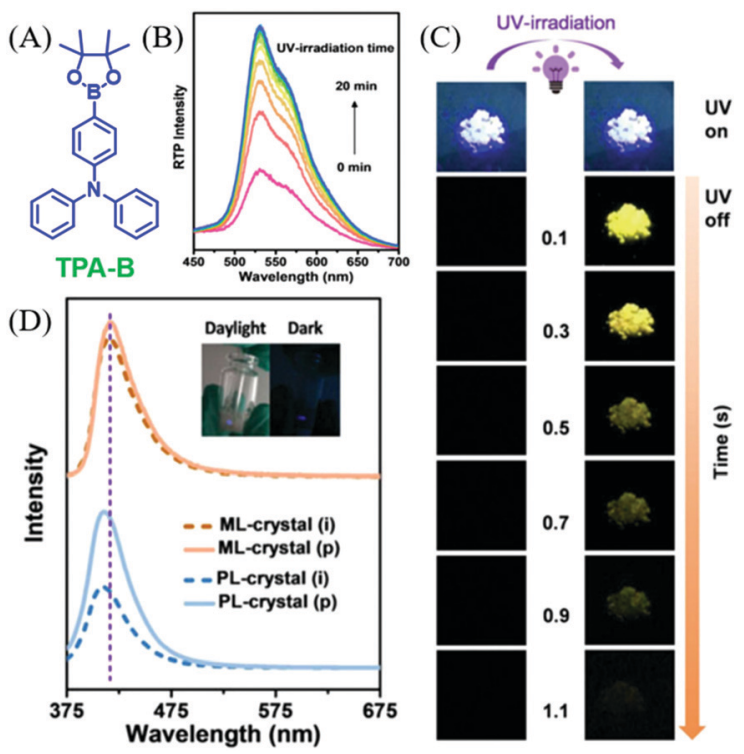

Fig. 22 (A) The molecular structure of TPA-B. (B) RTP spectra of TPA-B crystals under UV irradiation from 0 to $20 \mathrm{~min}$. (C) Photo-induced RTP behaviour of TPA-B crystals, including pictures of the fluorescence and afterglow for crystal (i) and crystal (p). (D) ML and PL spectra of TPA-B crystal in the initial state (crystal (i)) and after UV irradiation (crystal (p)). Inset: Photographs of TPA-B upon stirring with a glass rod in the daylight and in the dark. Reproduced with permission. ${ }^{100}$ Copyright 2018, John Wiley and Sons.

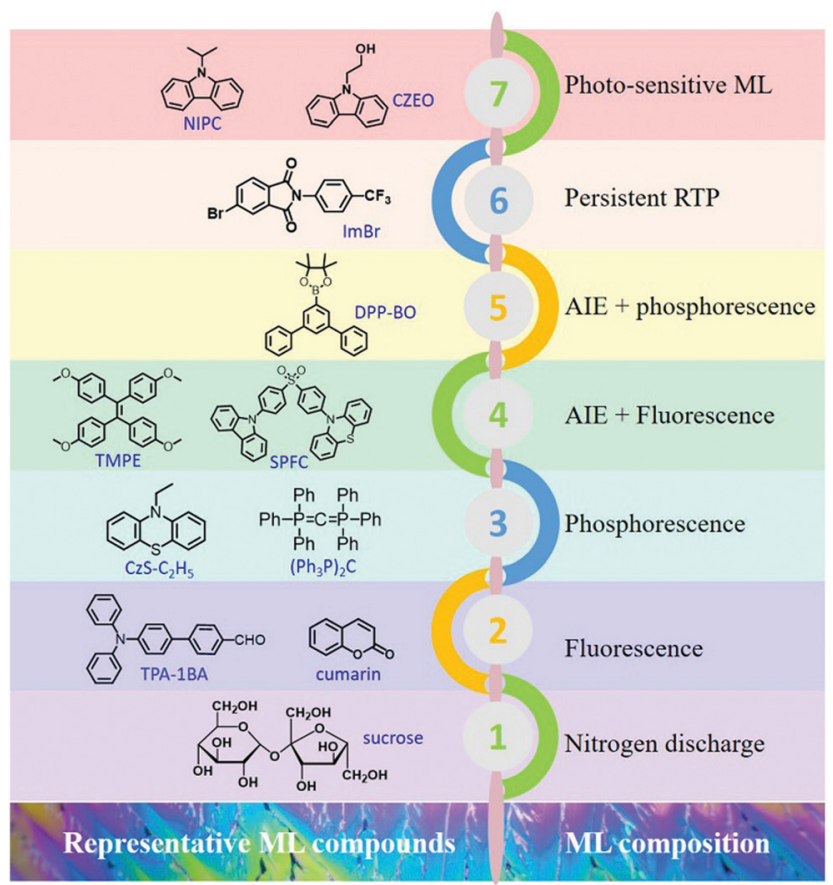

Fig. 23 Representative ML compounds and ML compositions during the different periods of development.

prohibited at $77 \mathrm{~K}$. The analysis of single crystals confirmed the subtle molecular motions and stronger intermolecular interactions after the photoinduction process. 


\section{Summary and outlook}

In summary, organic ML materials have achieved breakthrough developments in recent years, and more characteristics are being discovered from different aspects. It has been recognized that the luminous properties in the aggregated state could be analyzed with the consideration of the MUSIC concept; in other words, the luminous character is highly dependent on the molecular configuration and intermolecular packing style in the aggregate state. The representative ML compounds and ML compositions at different periods of development are listed in Fig. 23. ML has been discovered in different kinds of organic compounds, such as the traditional aromatic compounds, AIE compounds, TADF compounds, etc. ${ }^{101-103}$ The ML composition is also becoming more complicated, from the initial stage of nitrogen discharge, to the fluorescence, to the dual emission of fluorescence and phosphorescence, and the novel RTP, and photo-sensitive ML. The mechanical stimuli serve as the excitation source, and ML is the peculiar characteristic of molecules in the aggregated state, and more ML features emerge during the research progress. More importantly, the investigation of ML will broaden and deepen the understanding of the aggregate-state chemistry, and promote the development of the luminescence theory in the solid-state. However, it should be acknowledged that the study on organic ML is still in the initial stages, and the systemic theories on the characteristic of organic ML compounds are rare. Although some structural features have been gradually presented, the reported organic ML compounds are still limited to independent cases. On the other hand, the application research of organic ML is scarce, which may limit further development.

\section{Conflicts of interest}

There are no conflicts to declare.

\section{Acknowledgements}

This work was supported by the Starting Grants of Tianjin University and Tianjin Government.

\section{Notes and references}

1 Z. Yang, Z. Mao, Z. Xie, Y. Zhang, S. Liu, J. Zhao, J. Xu, Z. Chi and M. P. Aldred, Chem. Soc. Rev., 2017, 46, 915-1016.

2 T. Yu, L. Liu, Z. Xie and Y. Ma, Sci. China: Chem., 2015, 58, 907-915.

3 Y. Im, M. Kim, Y. J. Cho, J.-A. Seo, K. S. Yook and J. Y. Lee, Chem. Mater., 2017, 29, 1946-1963.

4 Q. Li and Z. Li, Adv. Sci., 2017, 4, 1600484.

5 K. Li and B. Liu, Chem. Soc. Rev., 2014, 43, 6570-6597.

6 B. Yang, J. Xiao, J. I. Wong, J. Guo, Y. Wu, L. Ong, L. L. Lao, F. Boey, H. Zhang, H. Y. Yang and Q. Zhang, J. Phys. Chem. C, 2011, 115, 7924-7927.
7 N. J. Turro, V. Ramamurth and J. C. Scaiano, Principles of molecular photochemistry: an introduction, University Science Books, Sausalito, California, 2009.

8 S. Hirata, Y. Sakai, K. Masui, H. Tanaka, S. Y. Lee, H. Nomura, N. Nakamura, M. Yasumatsu, H. Nakanotani, Q. Zhang, K. Shizu, H. Miyazaki and C. Adachi, Nat. Mater., 2015, 14, 330-336.

9 H. Uoyama, K. Goushi, K. Shizu, H. Nomura and C. Adachi, Nature, 2012, 492, 234-238.

10 M. K. Etherington, F. Franchello, J. Gibson, T. Northey, J. Santos, J. S. Ward, H. F. Higginbotham, P. Data, A. Kurowska, P. L. Dos Santos, D. R. Graves, A. S. Batsanov, F. B. Dias, M. R. Bryce, T. J. Penfold and A. P. Monkman, Nat. Commun., 2017, 8, 14987.

11 Y. Pan, W. Li, S. Zhang, L. Yao, C. Gu, H. Xu, B. Yang and Y. Ma, Adv. Opt. Mater., 2014, 2, 510-515.

12 Q. Peng, W. Li, S. Zhang, P. Chen, F. Li and Y. Ma, Adv. Opt. Mater., 2013, 1, 362-366.

13 Y. Deng, W. Yuan, Z. Jia and G. Liu, J. Phys. Chem. B, 2014, 118, 14536-14545.

14 J. Luo, Z. Xie, J. W. Y. Lam, L. Cheng, B. Z. Tang, H. Chen, C. Qiu, H. S. Kwok, X. Zhan, Y. Liu and D. Zhu, Chem. Commun., 2001, 1740-1741.

15 X. Chen, W. Luo, H. Ma, Q. Peng, W. Z. Yuan and Y. Zhang, Sci. China: Chem., 2018, 61, 351-359.

16 Y. Huang, Z. Wang, Z. Chen and Q. Zhang, Angew. Chem., Int. Ed., 2019, 58, 9696-9711.

17 H. Ye, G. Liu, S. Liu, D. Casanova, X. Ye, X. Tao, Q. Zhang and Q. Xiong, Angew. Chem., Int. Ed., 2018, 57, 1928-1932.

18 Y. Huang, J. Xing, Q. Gong, L. C. Chen, G. Liu, C. Yao, Z. Wang, H. L. Zhang, Z. Chen and Q. Zhang, Nat. Commun., 2019, 10, 169.

19 Q. Li, Y. Tang, W. Hu and Z. Li, Small, 2018, 14, 1801560.

20 Y. Cao, V. Fatemi, S. Fang, K. Watanabe, T. Taniguchi, E. Kaxiras and P. Jarillo-Herrero, Nature, 2018, 556, 43-50.

21 Y. Cao, V. Fatemi, A. Demir, S. Fang, S. L. Tomarken, J. Y. Luo, J. D. Sanchez-Yamagishi, K. Watanabe, T. Taniguchi, E. Kaxiras, R. C. Ashoori and P. Jarillo-Herrero, Nature, 2018, 556, 80-84.

22 A. J. Walton, Adv. Phys., 1977, 26, 887-948.

23 G. E. Hardy and J. I. Zink, Inorg. Chem., 1976, 15, 3061-3065.

24 Y. Xie and Z. Li, Chem, 2018, 4, 943-971.

25 E. Ubba, T. Yu, Z. Yang, J. Zhao, L. Wang and Z. Chi, Chem. - Asian J., 2018, 13, 3106-3121.

26 S. Mukherjee and P. Thilagar, Angew. Chem., Int. Ed., 2019, 58, 2-13.

27 Dengfeng Peng, Bing Chen and F. Wang, ChemPlusChem, 2015, 80, 1209-1215.

28 G. E. Hardy, J. C. Baldwin, J. I. Zink, W. C. Kaska, P.-H. Liu and L. Duboisi, J. Am. Chem. Soc., 1977, 99, 3552-3558.

29 J. I. Zink, G. E. Hardy and J. E. Sutton, J. Phys. Chem., 1976, 80, 248-249.

30 I. Sage, R. Badcock, L. Humberstone, N. Geddes, M. Kemp and G. Bourhill, Smart Mater. Struct., 1999, 8, 504-510.

31 C. N. Xu, T. Watanabe, M. Akiyama and X. G. Zheng, Appl. Phys. Lett., 1999, 74, 1236-1238. 
32 N. Terasaki, H. Yamada and C.-N. Xu, Catal. Today, 2013, 201, 203-208.

33 S. M. Jeong, S. Song, K.-I. Joo, J. Kim, S.-H. Hwang, J. Jeong and H. Kim, Energy Environ. Sci., 2014, 7, 3338-3346.

34 N. Terasaki and C. N. Xu, J. Colloid Interface Sci., 2014, 427, 62-66.

35 X. Wang, H. Zhang, R. Yu, L. Dong, D. Peng, A. Zhang, Y. Zhang, H. Liu, C. Pan and Z. L. Wang, Adv. Mater., 2015, 27, 2324-2331.

36 J.-C. Zhang, Y.-Z. Long, X. Yan, X. Wang and F. Wang, Chem. Mater., 2016, 28, 4052-4057.

37 Y. Chen, Y. Zhang, D. Karnaushenko, L. Chen, J. Hao, F. Ding and O. G. Schmidt, Adv. Mater., 2017, 29, 1605165.

38 D. Tu, C. N. Xu, A. Yoshida, M. Fujihala, J. Hirotsu and X. G. Zheng, Adv. Mater., 2017, 29, 1606914.

39 Y. Yuan and Y.-L. Chen, Chin. J. Polym. Sci., 2017, 35, 1315-1327.

40 P. Jha and B. P. Chandra, Luminescence, 2014, 29, 977-993.

41 D. O. Olawale, T. Dickens, W. G. Sullivan, O. I. Okoli, J. O. Sobanjo and B. Wang, J. Lumin., 2011, 131, 1407-1418.

42 L. M. Sweeting and A. L. Rheingold, J. Am. Chem. Soc., 1987, 109, 2652-2658.

43 J. I. Zink and W. C. Kaska, J. Am. Chem. Soc., 1973, 95, 7510-7512.

44 B. P. Chandra and J. I. Zink, Inorg. Chem., 1980, 19, 3098-3102.

45 J. T. Dickinson, L. B. Brix and L. C. Jensen, J. Phys. Chem., 1984, 88, 1698-1701.

46 J. T. Dickinson and L. C. Jensen, J. Polym. Sci., 1985, 23, 873-888.

47 J. T. Dickinson, L. C. Jensen and S. K. Bhattacharya, J. Vac. Sci. Technol., A, 1985, 3, 1398-1402.

48 M. Liu, Q. Wu, H. Shi, Z. An and W. Huang, Acta Chim. Sin., 2018, 76, 246-258.

49 B. P. Chandra and M. Elyas, Krist. Tech., 1978, 13, 1341-1343.

50 Q. Sun, L. Tang, Z. Zhang, K. Zhang, Z. Xie, Z. Chi, H. Zhang and W. Yang, Chem. Commun., 2018, 54, 94-97.

51 M. Fang, J. Yang, Q. Liao, Y. Gong, Z. Xie, Z. Chi, Q. Peng, Q. Li and Z. Li, J. Mater. Chem. C, 2017, 5, 9879-9885.

52 Y. Yu, C. Wang, Y. Wei, Y. Fan, J. Yang, J. Wang, M. Han, Q. Li and Z. Li, Adv. Opt. Mater., 2019, 18, 1900505.

53 K. Zhang, Q. Sun, Z. Zhang, L. Tang, Z. Xie, Z. Chi, S. Xue, H. Zhang and W. Yang, Chem. Commun., 2018, 54, 5225-5228.

54 Q. Sun, K. Zhang, Z. Zhang, L. Tang, Z. Xie, Z. Chi, S. Xue, H. Zhang and W. Yang, Chem. Commun., 2018, 54, 8206-8209.

55 C. Sun, X. Ran, X. Wang, Z. Cheng, Q. Wu, S. Cai, L. Gu, N. Gan, H. Shi, Z. An, H. Shi and W. Huang, J. Phys. Chem. Lett., 2018, 9, 335-339.

56 R. Nowak, A. Krajewska and M. Samoc, Chem. Phys. Lett., 1982, 94, 270-271.

57 W. Wu, T. Narisawa and S. Hayashi, Jpn. J. Appl. Phys., 2001, 40, 1294-1296.

58 N. Kitamura, O. Saravari, H.-B. Kim and S. Tazuke, Chem. Phys. Lett., 1986, 152, 360-363.
59 W. Li, Q. Huang, Z. Mao, Q. Li, L. Jiang, Z. Xie, R. Xu, Z. Yang, J. Zhao, T. Yu, Y. Zhang, M. P. Aldred and Z. Chi, Angew. Chem., Int. Ed., 2018, 57, 12727-12732.

60 J. Mei, N. L. Leung, R. T. Kwok, J. W. Lam and B. Z. Tang, Chem. Rev., 2015, 115, 11718-11940.

61 Y. W. Wu, A. J. Qin and B. Z. Tang, Chin. J. Polym. Sci., 2016, 35, 141-154.

62 Y. Duan, X. Xiang and Y. Dong, Acta Chim. Sin., 2016, 74, 923-928.

63 X. Ye, Y. Liu, Y. Lv, G. Liu, X. Zheng, Q. Han, K. A. Jackson and X. Tao, Angew. Chem., Int. Ed., 2015, 54, 7976-7980.

64 Y. Xie, T. Zhang, Z. Li, Q. Peng, Y. Yi and Z. Shuai, Chem. Asian J., 2015, 10, 2154-2161.

65 W. Z. Yuan, Y. Gong, S. Chen, X. Y. Shen, J. W. Y. Lam, P. Lu, Y. Lu, Z. Wang, R. Hu, N. Xie, H. S. Kwok, Y. Zhang, J. Z. Sun and B. Z. Tang, Chem. Mater., 2012, 24, 1518-1528.

66 Y. Bai, D. Liu, Z. Han, Y. Chen, Z. Chen, Y. Jiao, W. He and Z. Guo, Sci. China: Chem., 2018, 61, 1413-1422.

67 S. Wang, F. Wang, C. Li, T. Li, D. Cao and X. Ma, Sci. China: Chem., 2018, 61, 1301-1306.

68 S. Xu, T. Liu, Y. Mu, Y. F. Wang, Z. Chi, C. C. Lo, S. Liu, Y. Zhang, A. Lien and J. Xu, Angew. Chem., Int. Ed., 2015, 54, 874-878.

69 J. Guo, X.-L. Li, H. Nie, W. Luo, S. Gan, S. Hu, R. Hu, A. Qin, Z. Zhao, S.-J. Su and B. Z. Tang, Adv. Funct. Mater., 2017, 27, 1606458.

70 J. W. Y. L. Zujin Zhao and B. Z. Tang, Curr. Org. Chem., 2010, 14, 2109-2132.

71 R. Hu, N. L. Leung and B. Z. Tang, Chem. Soc. Rev., 2014, 43, 4494-4562.

72 M. Gao and B. Z. Tang, ACS Sens., 2017, 2, 1382-1399.

73 J. Mei, Y. Hong, J. W. Lam, A. Qin, Y. Tang and B. Z. Tang, Adv. Mater., 2014, 26, 5429-5479.

74 Q. Xia, Z. Chen, Z. Zhang and R. Liu, Chin. J. Org. Chem., 2018, 38, 2700-2705.

75 B. Xu, W. Li, J. He, S. Wu, Q. Zhu, Z. Yang, Y.-C. Wu, Y. Zhang, C. Jin, P.-Y. Lu, Z. Chi, S. Liu, J. Xu and M. R. Bryce, Chem. Sci., 2016, 7, 5307-5312.

76 B. Xu, J. He, Y. Mu, Q. Zhu, S. Wu, Y. Wang, Y. Zhang, C. Jin, C. Lo, Z. Chi, A. Lien, S. Liu and J. Xu, Chem. Sci., 2015, 6, 3236-3241.

77 C. Wang, B. Xu, M. Li, Z. Chi, Y. Xie, Q. Li and Z. Li, Mater. Horiz., 2016, 3, 220-225.

78 Y. Xie, J. Tu, T. Zhang, J. Wang, Z. Xie, Z. Chi, Q. Peng and Z. Li, Chem. Commun., 2017, 53, 11330-11333.

79 F. Liu, J. Tu, X. Wang, J. Wang, Y. Gong, M. Han, X. Dang, Q. Liao, Q. Peng, Q. Li and Z. Li, Chem. Commun., 2018, 54, 5598-5601.

80 K. K. Neena, P. Sudhakar, K. Dipak and P. Thilagar, Chem. Commun., 2017, 53, 3641-3644.

81 C. Arivazhagan, A. Maity, K. Bakthavachalam, A. Jana, S. K. Panigrahi, E. Suresh, A. Das and S. Ghosh, Chem. Eur. J., 2017, 23, 7046-7051.

82 Y.-B. Gong, P. Zhang, Y.-R. Gu, J.-Q. Wang, M.-M. Han, C. Chen, X.-J. Zhan, Z.-L. Xie, B. Zou, Q. Peng, Z.-G. Chi and Z. Li, Adv. Opt. Mater., 2018, 6, 1800198. 
83 J. Yang, J. Qin, P. Geng, J. Wang, M. Fang and Z. Li, Angew. Chem., Int. Ed., 2018, 57, 14174-14178.

84 Z. Xie, T. Yu, J. Chen, E. Ubba, L. Wang, Z. Mao, T. Su, Y. Zhang, M. P. Aldred and Z. Chi, Chem. Sci., 2018, 9, 5787-5794.

85 Z. He, W. Zhao, J. W. Y. Lam, Q. Peng, H. Ma, G. Liang, Z. Shuai and B. Z. Tang, Nat. Commun., 2017, 8, 416.

86 Y. Xie, Y. Ge, Q. Peng, C. Li, Q. Li and Z. Li, Adv. Mater., 2017, 29, 1606829.

87 B. Zhou and D. Yan, Adv. Funct. Mater., 2019, 29, 1807599.

88 Z. Lin, R. Kabe, N. Nishimura, K. Jinnai and C. Adachi, Adv. Mater., 2018, 30, 1803713.

89 J. Yang, X. Zhen, B. Wang, X. Gao, Z. Ren, J. Wang, Y. Xie, J. Li, Q. Peng, K. Pu and Z. Li, Nat. Commun., 2018, 9, 840.

90 J. I. Zink, J. Am. Chem. Soc., 1974, 96, 6775-6777.

91 J. Yang, X. Gao, Z. Xie, Y. Gong, M. Fang, Q. Peng, Z. Chi and Z. Li, Angew. Chem., 2017, 56, 15299-15303.

92 J. Yang, X. Gao, Z. Xie, Y. Gong, M. Fang, Q. Peng, Z. Chi and Z. Li, Angew. Chem., Int. Ed., 2017, 56, 15299-15303.

93 V. C. Wakchaure, K. C. Ranjeesh, Goudappagouda, T. Das, K. Vanka, R. Gonnade and S. S. Babu, Chem. Commun., 2018, 54, 6028-6031.
94 J. Wang, C. Wang, Y. Gong, Q. Liao, M. Han, T. Jiang, Q. Dang, Y. Li, Q. Li and Z. Li, Angew. Chem., Int. Ed., 2018, 57, 16821-16826.

95 J. Nishida, H. Ohura, Y. Kita, H. Hasegawa, T. Kawase, N. Takada, H. Sato, Y. Sei and Y. Yamashita, J. Org. Chem., 2016, 81, 433-441.

96 H. Nakayama, J.-I. Nishida, N. Takada, H. Sato and Y. Yamashita, Chem. Mater., 2012, 24, 671-676.

97 J. A. Li, J. Zhou, Z. Mao, Z. Xie, Z. Yang, B. Xu, C. Liu, X. Chen, D. Ren, H. Pan, G. Shi, Y. Zhang and Z. Chi, Angew. Chem., 2018, 57, 6449-6453.

98 Y. Mu, Z. Yang, J. Chen, Z. Yang, W. Li, X. Tan, Z. Mao, T. Yu, J. Zhao, S. Zheng, S. Liu, Y. Zhang, Z. Chi, J. Xu and M. P. Aldred, Chem. Sci., 2018, 9, 3782-3787.

99 Q. Huang, X. Mei, Z. Xie, D. Wu, S. Yang, W. Gong, Z. Chi, Z. Lin and Q. Ling, J. Mater. Chem. C, 2019, 7, 2530-2534.

100 Q. Dang, L. Hu, J. Wang, Q. Zhang, M. Han, S. Luo, Y. Gong, C. Wang, Q. Li and Z. Li, Chem. - Eur. J., 2019, 25, 7031-7037.

101 J. Yang, Z. Chi, W. Zhu, B. Z. Tang and Z. Li, Sci. China: Chem., 2019, 62, 1090-1098.

102 Q. Li and Z. Li, Sci. China Mater., 2019, 62, DOI: 10.1007/ s40843-019-1172-2.

103 Y. Xie and Z. Li, Chem. - Asian J., 2019, 14, 2524-2541. 